\title{
LIMIT CYCLES \\ FOR A CLASS OF CONTINUOUS AND DISCONTINUOUS CUBIC POLYNOMIAL DIFFERENTIAL SYSTEMS
}

\author{
JAUME LLIBRE ${ }^{1}$, BRUNO D. LOPES ${ }^{1}$ AND JAIME R. DE MORAES ${ }^{2}$
}

\begin{abstract}
We study the maximum number of limit cycles that bifurcate from the periodic solutions of the family of isochronous cubic polynomial centers

$\dot{x}=y\left(-1+2 \alpha x+2 \beta x^{2}\right), \quad \dot{y}=x+\alpha\left(y^{2}-x^{2}\right)+2 \beta x y^{2}, \quad \alpha \in \mathbb{R}, \beta<0$, when it is perturbed inside the classes of all continuous and discontinuous cubic polynomial differential systems. We obtain that the maximum number of limit cycles which can be obtained by the averaging method of first order is 3 for the perturbed continuous systems and for the perturbed discontinuous systems at least 12 limit cycles can appear.
\end{abstract}

\section{Introduction and Statement of the Main Results}

One of the main open problems in the qualitative theory of real planar differential systems is the determination of their limit cycles. A classical way to produce limit cycles is perturbing a system which has a center. Thus the limit cycles bifurcate in the perturbed system from some of the periodic orbits of the period annulus of the center of the unperturbed system, see for instance Pontrjagin [22], the second part of the book [8] and the hundreds of references quoted there. In this paper we shall perturb isochronous centers. The perturbation of some of these centers have already been studied see for instance $[7,14]$. For a survey on isochronous centers see [6].

In [5] the authors studied some classes of isochronous cubic polynomial differential systems. In particular they obtained the family

$$
\begin{aligned}
& \dot{x}=y\left(-1+2 \alpha x+2 \beta x^{2}\right)=P(x, y), \\
& \dot{y}=x+\alpha\left(y^{2}-x^{2}\right)+2 \beta x y^{2}=Q(x, y),
\end{aligned}
$$

where $\alpha \in \mathbb{R}$ and $\beta<0$. This family has a rational first integral of degree 2 , see system (iv) of Theorem 7 in [5], see also [16, 17]. An open question is: What happens with the periodic orbits of the unperturb system (1) when it is perturbed inside the class of all cubic continuous and discontinuous polynomial differential systems? More precisely consider the following

2010 Mathematics Subject Classification. 34A36, 34C07, 34C25, 34C29.

Key words and phrases. polynomial vector field, limit cycle, averaging method, periodic orbit, isochronous center. 
systems

$$
\begin{aligned}
& \dot{x}=y\left(-1+2 \alpha x+2 \beta x^{2}\right)+\varepsilon p_{1}(x, y), \\
& \dot{y}=x+\alpha\left(y^{2}-x^{2}\right)+2 \beta x y^{2}+\varepsilon q_{1}(x, y),
\end{aligned}
$$

and the discontinuous system

$$
\left(\begin{array}{c}
\dot{x} \\
\dot{y}
\end{array}\right)=\left\{\begin{array}{lll}
X_{1}(x, y) & \text { if } \quad y>0 \\
X_{2}(x, y) & \text { if } \quad y<0
\end{array}\right.
$$

where

$$
\begin{aligned}
& X_{1}(x, y)=\left(\begin{array}{c}
y\left(-1+2 \alpha x+2 \beta x^{2}\right)+\varepsilon p_{1}(x, y) \\
x+\alpha\left(y^{2}-x^{2}\right)+2 \beta x y^{2}+\varepsilon q_{1}(x, y)
\end{array}\right), \\
& X_{2}(x, y)=\left(\begin{array}{c}
y\left(-1+2 \alpha x+2 \beta x^{2}\right)+\varepsilon p_{2}(x, y) \\
x+\alpha\left(y^{2}-x^{2}\right)+2 \beta x y^{2}+\varepsilon q_{2}(x, y)
\end{array}\right),
\end{aligned}
$$

with

$$
\begin{aligned}
& p_{1}(x, y)=a_{1} x+a_{2} y+a_{3} x^{2}+a_{4} x y+a_{5} y^{2}+a_{6} x^{3}+a_{7} x^{2} y+a_{8} x y^{2}+a_{9} y^{3}, \\
& q_{1}(x, y)=b_{1} x+b_{2} y+b_{3} x^{2}+b_{4} x y+b_{5} y^{2}+b_{6} x^{3}+b_{7} x^{2} y+b_{8} x y^{2}+b_{9} y^{3}, \\
& p_{2}(x, y)=c_{1} x+c_{2} y+c_{3} x^{2}+c_{4} x y+c_{5} y^{2}+c_{6} x^{3}+c_{7} x^{2} y+c_{8} x y^{2}+c_{9} y^{3}, \\
& q_{2}(x, y)=d_{1} x+d_{2} y+d_{3} x^{2}+d_{4} x y+d_{5} y^{2}+d_{6} x^{3}+d_{7} x^{2} y+d_{8} x y^{2}+d_{9} y^{3} .
\end{aligned}
$$

In this paper we study the maximum number of limit cycles of systems (2) and (3) which can be obtained using the averaging theory of first order.

Essentially there are four methods for determining the number of limit cycles which bifurcate from the periodic orbits of a period annulus of a center. The first method is based in the Poincaré return map, see for instance $[2,7]$. The second method uses the Poincaré-Pontrjagin-Melnikov integrals or the Abelian integrals. These two methods are equivalent in the plane, see section 6 of Chapter 4 of [12] and section 5 of Chapter 6 of [1]. The third method is based on the inverse integrating factor, see section 6 of [9] or $[10,11,25]$. The last method is based on the averaging theory, see for example $[3,23,24]$. From [3] it is easy to check that in the plane the averaging method of first order is equivalent to the method of the Abelian integrals. Moreover the first two methods only give information on the number of periodic orbits of the unperturbed system that become limit cycles after the perturbation. The last two methods also can give the shape of the bifurcated limit cycle up to the order of the perturbation parameter, see $[10,11,15]$.

In what follows we state our main results.

Theorem 1. For $|\varepsilon| \neq 0$ sufficiently small the maximum number of limit cycles of system (2), bifurcating from the periodic solutions of isochronous center (1), is at most 3 using the averaging theory of first order, and this number is reached.

Theorem 1 is proved in Section 3. 
Theorem 2. For $|\varepsilon| \neq 0$ sufficiently small the maximum number of limit cycles of system (3), bifurcating from the periodic solutions of isochronous center (1), is at least 12 using the averaging theory of first order.

Theorem 2 is proved in Section 4.

In Section 2 we present the basic results that we need for proving Theorems 1 and 2 .

\section{Preliminaries}

In this section we give some known results that we shall need for proving our results. The following theorem provides periodic solutions of a periodic continous differential system. See [24] for a proof.

Consider the differential equation

$$
\dot{x}=\varepsilon F(t, x)+\varepsilon^{2} R(t, x, \varepsilon), \quad x(0)=x_{0},
$$

with $x \in D$, where $D$ is an open subset of $\mathbb{R}^{n}$, and $t \geq 0$. Moreover we assume that $F(t, x)$ is $T$-periodic in $t$. Separately, we consider in $D$ the averaged differential equation

$$
\dot{y}=\varepsilon f(y), \quad y(0)=x_{0},
$$

where

$$
f(y)=\frac{1}{T} \int_{0}^{T} F(t, y) d t .
$$

Theorem 3. Consider the two initial value problems (4) and (5). Suppose:

(i) $F$, its Jacobian $\partial F / \partial x$, its Hessian $\partial^{2} F / \partial x^{2}$ are defined, continuous and bounded by an independent constant of $\varepsilon$ in $[0, \infty) \times D$ and $\varepsilon \in\left(0, \varepsilon_{0}\right]$.

(ii) $F$ is $T$-periodic in $t$ ( $T$ independent of $\varepsilon$ ).

(iii) $y(t)$ belongs to $D$ on the interval of time $[0,1 / \varepsilon]$.

Then the following statements hold.

(a) For $t \in[0,1 / \varepsilon]$ we have that $x(t)-y(t)=\mathcal{O}(\varepsilon)$ as $\varepsilon \rightarrow 0$.

(b) If $p$ is an equilibrium point of the averaged equation (5) and

$$
\left.\operatorname{det}\left(\frac{\partial f}{\partial y}\right)\right|_{y=p} \neq 0
$$

then there exists a T-periodic solution $x(t, \varepsilon)$ of equation (4) such that $x(0, \varepsilon) \rightarrow p$ as $\varepsilon \rightarrow 0$.

(c) The stability or instability of the periodic solution $x(t, \varepsilon)$ is given by the stability or instability of the equilibrium point $p$ of the averaged system (5). In fact the equilibrium point $p$ has the stability behavior of the Poincaré map associated to the periodic solution $x(t, \varepsilon)$.

The following theorem is a discontinuous version of previous theorem which provides periodic solutions of a periodic discontinuous differential system. See [18] for a proof. 
Theorem 4. Consider the following discontinuous differential system

$$
\dot{x}(t)=\varepsilon F(t, x)+\varepsilon^{2} R(t, x, \varepsilon),
$$

with

$$
\begin{aligned}
F(t, x) & =F_{1}(t, x)+\operatorname{sign}(h(t, x)) F_{2}(t, x), \\
R(t, x, \varepsilon) & =R_{1}(t, x, \varepsilon)+\operatorname{sign}(h(t, x)) R_{2}(t, x, \varepsilon),
\end{aligned}
$$

where $F_{1}, F_{2}: \mathbb{R} \times D \rightarrow \mathbb{R}^{n}, R_{1}, R_{2}: \mathbb{R} \times D \times\left(-\varepsilon_{0}, \varepsilon_{0}\right) \rightarrow \mathbb{R}^{n}$ and $h:$ $\mathbb{R} \times D \rightarrow \mathbb{R}$ are continuous functions, T-periodic in the variable $t$ and $D$ is an open subset of $\mathbb{R}^{n}$. We also suppose that $h$ is a $C^{1}$ function having 0 as a regular value. Denote by $\mathcal{M}=h^{-1}(0)$, by $\Sigma=\{0\} \times D \nsubseteq \mathcal{M}$, by $\Sigma_{0}=\Sigma \backslash \mathcal{M} \neq \emptyset$ and its elements by $z \equiv(0, z) \notin \mathcal{M}$.

Define the averaged function $f: D \rightarrow \mathbb{R}^{n}$ as

$$
f(x)=\int_{0}^{T} F(t, x) d t .
$$

Assume the following three conditions.

(i) $F_{1}, F_{2}, R_{1}, R_{2}$ and $h$ are locally $L$-Lipschitz with respect to $x$.

(ii) For $a \in \Sigma_{0}$ with $f(a)=0$ there exist a neighborhood $V$ of a such that $f(z) \neq 0$ for all $z \in \bar{V} \backslash\{a\}$ and $d_{B}(f, V, a) \neq 0$, (i.e. the Brouwer degree of $f$ at a is not zero).

(iii) If $(\partial h / \partial t)(t, z) \not \equiv 0$ then for all $\left(t_{0}, z_{0}\right) \in \mathcal{M}$ we have $(\partial h / \partial t)\left(t_{0}, z_{0}\right) \neq$ 0. If $(\partial h / \partial t)\left(t_{0}, z_{0}\right) \equiv 0$ then $\left(\left\langle\nabla_{x} h, F_{1}\right\rangle^{2}-\left\langle\nabla_{x} h, F_{2}\right\rangle^{2}\right)\left(t_{0}, z_{0}\right)>0$, for all $\left(t_{0}, z_{0}\right) \in[0, T] \times \mathcal{M}$.

Then for $|\varepsilon|>0$ sufficiently small there exists a $T$-periodic solution $x(\cdot, \varepsilon)$ of system (6) such that $x(t, \varepsilon) \rightarrow a$ as $\varepsilon \rightarrow 0$.

If the function $f$ of Theorem 4 is of class $C^{1}$, then it is sufficient to see that the Jacobian of the function $f$ evaluated at $a$ is non-zero for showing that $d_{B}(f, V, a) \neq 0$. For more details see Theorem 1.1.2 of [21].

Consider a planar system

$$
\dot{x}=P(x, y), \quad \dot{y}=Q(x, y),
$$

where $P, Q: \mathbb{R}^{2} \rightarrow \mathbb{R}$ are continuous functions. Suppose that system (7) has a continuous family of ovals

$$
\left\{\Gamma_{h}\right\} \subset\left\{(x, y): H(x, y)=h, h_{1}<h<h_{2}\right\}
$$

where $H$ is a first integral of (7). Consider the following perturbations of system (7)

$$
\dot{x}=P(x, y)+\varepsilon p(x, y), \quad \dot{y}=Q(x, y)+\varepsilon q(x, y),
$$

where $p, q: \mathbb{R}^{2} \rightarrow \mathbb{R}$ are continuous functions.

The next theorem (see Theorem 5.2 of [3] for a proof) provides a tool for transforming the perturbed system (8) in the standard form of the averaging theory given in Theorem 3 . 
Theorem 5. Consider system (7) and its first integral $H$. Assume that $x Q(x, y)-y P(x, y) \neq 0$ for all $(x, y)$ in the period annulus formed by the ovals $\left\{\Gamma_{h}\right\}$. Let $\rho:\left(\sqrt{h_{1}}, \sqrt{h_{2}}\right) \times[0,2 \pi) \rightarrow[0, \infty)$ be a continuous function such that

$$
H(\rho(R, \varphi) \cos \varphi, \rho(R, \varphi) \sin \varphi)=R^{2},
$$

for all $R \in\left(\sqrt{h_{1}}, \sqrt{h_{2}}\right)$ and all $\varphi \in[0,2 \pi)$. Then the differential equation which describes the dependence between the square root of the energy $R=\sqrt{h}$ and the angle $\varphi$ for system (8) is

$$
\frac{d R}{d \varphi}=\varepsilon \frac{\mu\left(x^{2}+y^{2}\right)(Q p-P q)}{2 R(Q x-P y)}+\mathcal{O}\left(\varepsilon^{2}\right)
$$

where $\mu=\mu(x, y)$ is the integrating factor of system (7) corresponding to the first integral $H, x=\rho(R, \varphi) \cos \varphi$ and $y=\rho(R, \varphi) \sin \varphi$.

We recall that $\mu$ is the integrating factor corresponding to the first integral $H$ of system (7) if

$$
\mu P=-\frac{\partial H}{\partial y} \quad \text { and } \quad \mu Q=\frac{\partial H}{\partial x} .
$$

Let $I$ be a real interval and let $f_{0}, \ldots, f_{n}: I \rightarrow \mathbb{R}$ be functions. We say that $f_{0}, \ldots, f_{n}$ are linearly independent functions if and only if

$$
\forall s \in I \quad \sum_{i=0}^{n} \lambda_{i} f_{i}(s)=0 \quad \Rightarrow \quad \lambda_{0}=\lambda_{1}=\ldots=\lambda_{n}=0 .
$$

The next result well-known can be found in Proposition 1 of [19].

Proposition 6. If $f_{0}, \ldots, f_{n}$ are linearly independent then there exist $s_{1}, s_{2}, \ldots$, $s_{n} \in I$ and $\lambda_{0}, . ., \lambda_{n} \in \mathbb{R}$ such that for every $j \in\{1, \ldots, n\}$ we have

$$
\sum_{i=0}^{n} \lambda_{i} f_{i}\left(s_{j}\right)=0
$$

The functions $\left(f_{0}, f_{1}, \ldots, f_{n}\right)$ defined on $I$ form an Extended Chebyshev system or ET-system on $I$, if and only if any nontrivial linear combination of these functions has at most $n$ zeros counting their multiplicities and this number is reached. We say that $F$ is an Extended Complete Chebyshev system or an ECT-system on $I$ if and only if for any $k \in\{0,1, \ldots, n\}$, $\left(f_{0}, f_{1}, \ldots, f_{k}\right)$ form an ET-system. For proving that $\left(f_{0}, f_{1}, \ldots, f_{k}\right)$ is an ECT-system on $I$ is sufficient and necessary to prove that $W\left(f_{0}, \ldots, f_{k}\right)(s) \neq$ 0 on $I$ for $k \in\{0,1, \ldots, n\}$. We denote by $W\left(f_{0}, \ldots, f_{k}\right)(s)$ the Wronskian of the functions $\left(f_{0}, \ldots, f_{k}\right)$ with respect to $s$. We remember that the definition of the Wronskian is

$$
W\left(f_{0}, \ldots, f_{k}\right)(s)=\left|\begin{array}{cccc}
f_{0}(s) & f_{1}(s) & \cdots & f_{k}(s) \\
f_{0}^{\prime}(s) & f_{1}^{\prime}(s) & \cdots & f_{k}^{\prime}(s) \\
\vdots & \vdots & \ddots & \vdots \\
f_{0}^{(k)}(s) & f_{1}^{(k)}(s) & \cdots & f_{k}^{(k)}(s)
\end{array}\right|
$$


For more details on ECT-system see [13].

\section{Proof of Theorem 1}

We note that the integrating factor

$$
\mu(x, y)=-\frac{2}{\left(-1+2 \alpha x+2 \beta x^{2}\right)^{2}}
$$

and the first integral

$$
H(x, y)=\frac{x^{2}+y^{2}}{1-2 x(\alpha+\beta x)}
$$

of system (1) satisfy $\mu P=-H_{y}$ and $\mu Q=H_{x}$. So $\mu$ is the integrate factor corresponding to the first integral $H$. The function $\rho:(0, \sqrt{-1 /(2 \beta})) \times$ $[0,2 \pi) \rightarrow[0, \infty)$ given by

$$
\rho(R, \varphi)=\frac{R\left(\sqrt{\left(\alpha^{2}+2 \beta\right) R^{2} \cos ^{2} \varphi+1}-\alpha R \cos \varphi\right)}{2 \beta R^{2} \cos ^{2} \varphi+1}
$$

satisfies hypothesis (9) of Theorem 5 . We shall see later on that $x Q(x, y)-$ $y P(x, y) \neq 0$ in the period annulus. Thus system (10) of Theorem 5 for our system (2) becomes

$$
\frac{d R}{d \varphi}=\varepsilon \sum_{i=1}^{11} \frac{A_{i}(\varphi, \alpha, \beta, a, b)}{B(R, \varphi, \alpha, \beta)} R^{i}+\mathcal{O}\left(\varepsilon^{2}\right)
$$

where $a=\left(a_{1}, \ldots, a_{9}\right), b=\left(b_{1}, \ldots, b_{9}\right)$ and

$$
\begin{aligned}
A_{1}= & a_{1} \cos ^{2} \varphi+\left(a_{2}+b_{1}\right) \sin \varphi \cos \varphi+b_{2} \sin ^{2} \varphi \\
A_{2}= & \sqrt{\left(\alpha^{2}+2 \beta\right) R^{2} \cos ^{2} \varphi+1}\left(\left(a_{3}-3 \alpha a_{1}\right) \cos ^{3} \varphi+\left(-3 \alpha a_{2}+a_{4}\right.\right. \\
& \left.-4 \alpha b_{1}+b_{3}\right) \sin \varphi \cos ^{2} \varphi+\left(a_{5}+\alpha\left(a_{1}-4 b_{2}\right)+b_{4}\right) \sin ^{2} \varphi \cos \varphi \\
& \left.+\left(\alpha a_{2}+b_{5}\right) \sin ^{3} \varphi\right), \\
A_{3}= & \left(5 a_{1} \alpha^{2}-4 a_{3} \alpha+a_{6}+10 a_{1} \beta\right) \cos ^{4} \varphi+\left(\left(5 a_{2}+8 b_{1}\right) \alpha^{2}-\left(4 a_{4}\right.\right. \\
& \left.\left.+5 b_{3}\right) \alpha+a_{7}+10 a_{2} \beta+8 \beta b_{1}+b_{6}\right) \sin \varphi \cos ^{3} \varphi+\left(\left(8 b_{2}-3 a_{1}\right) \alpha^{2}\right. \\
& \left.+\left(a_{3}-4 a_{5}-5 b_{4}\right) \alpha+a_{8}+2 a_{1} \beta+8 \beta b_{2}+b_{7}\right) \sin ^{2} \varphi \cos ^{2} \varphi \\
& +\left(-3 a_{2} \alpha^{2}+\left(a_{4}-5 b_{5}\right) \alpha+a_{9}+2 a_{2} \beta+b_{8}\right) \sin ^{3} \varphi \cos \varphi \\
& +\left(\alpha a_{5}+b_{9}\right) \sin ^{4} \varphi,
\end{aligned}
$$




$$
\begin{aligned}
& A_{4}=\sqrt{\left(\alpha^{2}+2 \beta\right) R^{2} \cos ^{2} \varphi+1}\left(\left(-4 a_{1} \alpha^{3}+8 a_{3} \alpha^{2}-5 a_{6} \alpha-24 a_{1} \beta \alpha\right.\right. \\
& \left.+8 a_{3} \beta\right) \cos ^{5} \varphi-\left(4\left(a_{2}+2 b_{1}\right) \alpha^{3}-4\left(2 a_{4}+3 b_{3}\right) \alpha^{2}+\left(5 a_{7}+6\left(4 a_{2} \beta\right.\right.\right. \\
& \left.\left.\left.+4 b_{1} \beta+b_{6}\right)\right) \alpha-2 \beta\left(4 a_{4}+3 b_{3}\right)\right) \sin \varphi \cos ^{4} \varphi+\left(4\left(a_{1}-2 b_{2}\right) \alpha^{3}\right. \\
& -4\left(a_{3}-2 a_{5}-3 b_{4}\right) \alpha^{2}+\left(a_{6}-5 a_{8}-6\left(4 \beta b_{2}+b_{7}\right)\right) \alpha+2 \beta\left(a_{3}+4 a_{5}\right. \\
& \left.\left.+3 b_{4}\right)\right) \sin ^{2} \varphi \cos ^{3} \varphi+\left(4 a_{2} \alpha^{3}-4\left(a_{4}-3 b_{5}\right) \alpha^{2}+\left(a_{7}-5 a_{9}\right.\right. \\
& \left.\left.+6 b_{8}\right) \alpha+2 \beta\left(a_{4}+3 b_{5}\right)\right) \sin ^{3} \varphi \cos ^{2} \varphi+\left(-4 a_{5} \alpha^{2}+\left(a_{8}-6 b_{9}\right) \alpha\right. \\
& \left.\left.+2 a_{5} \beta\right) \sin ^{4} \varphi \cos \varphi+\alpha a_{9} \sin ^{5} \varphi\right), \\
& A_{5}=\left(4 a_{1} \alpha^{4}-12 a_{3} \alpha^{3}+13 a_{6} \alpha^{2}+40 a_{1} \beta \alpha^{2}-32 a_{3} \beta \alpha+40 a_{1} \beta^{2}\right. \\
& \left.+8 a_{6} \beta\right) \cos ^{6} \varphi+\left(4 a_{2} \alpha^{4}+8 b_{1} \alpha^{4}-12 a_{4} \alpha^{3}-20 b_{3} \alpha^{3}+13 a_{7} \alpha^{2}\right. \\
& +40 a_{2} \beta \alpha^{2}+48 \beta b_{1} \alpha^{2}+18 b_{6} \alpha^{2}-32 a_{4} \beta \alpha-30 \beta b_{3} \alpha+40 a_{2} \beta^{2} \\
& \left.+8 a_{7} \beta+24 \beta^{2} b_{1}+6 \beta b_{6}\right) \sin \varphi \cos ^{5} \varphi+\left(-4 a_{1} \alpha^{4}+8 b_{2} \alpha^{4}+8 a_{3} \alpha^{3}\right. \\
& +12 a_{5} \alpha^{3}-20 b_{4} \alpha^{3}-5 a_{6} \alpha^{2}+13 a_{8} \alpha^{2}-8 a_{1} \beta \alpha^{2}+48 \beta b_{2} \alpha^{2} \\
& +18 b_{7} \alpha^{2}-2 a_{3} \beta \alpha-32 a_{5} \beta \alpha-30 \beta b_{4} \alpha+16 a_{1} \beta^{2}+2 a_{6} \beta+8 a_{8} \beta \\
& \left.+24 \beta^{2} b_{2}+6 \beta b_{7}\right) \sin ^{2} \varphi \cos ^{4} \varphi+\left(-4 a_{2} \alpha^{4}+8 a_{4} \alpha^{3}-20 b_{5} \alpha^{3}\right. \\
& -5 a_{7} \alpha^{2}+13 a_{9} \alpha^{2}-8 a_{2} \beta \alpha^{2}+18 b_{8} \alpha^{2}-2 a_{4} \beta \alpha-30 \beta b_{5} \alpha+16 a_{2} \beta^{2} \\
& \left.+2 a_{7} \beta+8 a_{9} \beta+6 \beta b_{8}\right) \sin ^{3} \varphi \cos ^{3} \varphi+\left(8 a_{5} \alpha^{3}-5 a_{8} \alpha^{2}+18 b_{9} \alpha^{2}\right. \\
& \left.-2 a_{5} \beta \alpha+2 a_{8} \beta+6 \beta b_{9}\right) \sin ^{4} \varphi \cos ^{2} \varphi-a_{9}\left(5 \alpha^{2}-2 \beta\right) \sin ^{5} \varphi \cos \varphi \text {, } \\
& A_{6}=2 \cos ^{2} \varphi \sqrt{\left(\alpha^{2}+2 \beta\right) R^{2} \cos ^{2} \varphi+1}\left(\left(4 a_{3} \alpha^{4}-2\left(5 a_{6}+6 a_{1} \beta\right) \alpha^{3}\right.\right. \\
& \left.+24 a_{3} \beta \alpha^{2}-3 \beta\left(5 a_{6}+12 a_{1} \beta\right) \alpha+12 a_{3} \beta^{2}\right) \cos ^{5} \varphi+\left(4 \left(a_{4}\right.\right. \\
& \left.+2 b_{3}\right) \alpha^{4}-2\left(5 a_{7}+6 a_{2} \beta+8 \beta b_{1}+8 b_{6}\right) \alpha^{3}+24 \beta\left(a_{4}+b_{3}\right) \alpha^{2} \\
& \left.-3 \beta\left(5 a_{7}+4\left(3 a_{2} \beta+2 b_{1} \beta+b_{6}\right)\right) \alpha+6 \beta^{2}\left(2 a_{4}+b_{3}\right)\right) \sin \varphi \cos ^{4} \varphi \\
& -\left(4\left(a_{3}-a_{5}-2 b_{4}\right) \alpha^{4}-2\left(3 a_{6}-5 a_{8}+2 a_{1} \beta-8 \beta b_{2}-8 b_{7}\right) \alpha^{3}\right. \\
& -24 \beta\left(a_{5}+b_{4}\right) \alpha^{2}+3 \beta\left(a_{6}+5 a_{8}+4 a_{1} \beta+8 \beta b_{2}+4 b_{7}\right) \alpha-6 \beta^{2}\left(a_{3}\right. \\
& \left.\left.+2 a_{5}+b_{4}\right)\right) \sin ^{2} \varphi \cos ^{3} \varphi-\left(4\left(a_{4}-2 b_{5}\right) \alpha^{4}-2\left(3 a_{7}-5 a_{9}+2 a_{2} \beta\right.\right. \\
& \left.-8 b_{8}\right) \alpha^{3}-24 \beta b_{5} \alpha^{2}+3 \beta\left(a_{7}+5 a_{9}+4 a_{2} \beta+4 b_{8}\right) \alpha \\
& -6 \beta^{2}\left(a_{4}+b_{5}\right) \sin ^{3} \varphi \cos ^{2} \varphi-\left(4 a_{5} \alpha^{4}+\left(16 b_{9}-6 a_{8}\right) \alpha^{3}+3 \beta\left(a_{8}\right.\right. \\
& \left.\left.\left.+4 b_{9}\right) \alpha-6 a_{5} \beta^{2}\right) \sin ^{4} \varphi \cos \varphi+3 \alpha a_{9}\left(2 \alpha^{2}-\beta\right) \sin ^{5} \varphi\right), \\
& A_{7}=2 \cos ^{3} \varphi\left(\left(-4 a_{3} \alpha^{5}+2\left(7 a_{6}+6 a_{1} \beta\right) \alpha^{4}-36 a_{3} \beta \alpha^{3}+3 \beta\left(13 a_{6}\right.\right.\right. \\
& \left.\left.+20 a_{1} \beta\right) \alpha^{2}-48 a_{3} \beta^{2} \alpha+4 \beta^{2}\left(3 a_{6}+10 a_{1} \beta\right)\right) \cos ^{5} \varphi-\left(4 \left(a_{4}\right.\right. \\
& \left.+2 b_{3}\right) \alpha^{5}-2\left(7 a_{7}+6 a_{2} \beta+8 \beta b_{1}+12 b_{6}\right) \alpha^{4}+4 \beta\left(9 a_{4}+10 b_{3}\right) \alpha^{3} \\
& -3 \beta\left(13 a_{7}+4\left(5 a_{2} \beta+4 b_{1} \beta+3 b_{6}\right)\right) \alpha^{2}+6 \beta^{2}\left(8 a_{4}+5 b_{3}\right) \alpha-2 \beta^{2}\left(6 a_{7}\right. \\
& \left.\left.+20 a_{2} \beta+8 \beta b_{1}+3 b_{6}\right)\right) \sin \varphi \cos ^{4} \varphi+\left(4\left(a_{3}-a_{5}-2 b_{4}\right) \alpha^{5}-2\left(5 a_{6}\right.\right. \\
& \left.-7 a_{8}+2 a_{1} \beta-8 \beta b_{2}-12 b_{7}\right) \alpha^{4}+4 \beta\left(a_{3}-9 a_{5}-10 b_{4}\right) \alpha^{3}+3 \beta\left(a_{6}\right. \\
& \left.+13 a_{8}+4 a_{1} \beta+16 \beta b_{2}+12 b_{7}\right) \alpha^{2}-6 \beta^{2}\left(3 a_{3}+8 a_{5}+5 b_{4}\right) \alpha
\end{aligned}
$$




$$
\begin{aligned}
& \left.+2 \beta^{2}\left(3 a_{6}+6 a_{8}+12 a_{1} \beta+8 \beta b_{2}+3 b_{7}\right)\right) \sin ^{2} \varphi \cos ^{3} \varphi+\left(4 \left(a_{4}\right.\right. \\
& \left.-2 b_{5}\right) \alpha^{5}-2\left(5 a_{7}-7 a_{9}+2 a_{2} \beta-12 b_{8}\right) \alpha^{4}+4 \beta\left(a_{4}-10 b_{5}\right) \alpha^{3} \\
& +3 \beta\left(a_{7}+13 a_{9}+4 a_{2} \beta+12 b_{8}\right) \alpha^{2}-6 \beta^{2}\left(3 a_{4}+5 b_{5}\right) \alpha+6 \beta^{2}\left(a_{7}\right. \\
& \left.\left.+2 a_{9}+4 a_{2} \beta+b_{8}\right)\right) \sin ^{3} \varphi \cos ^{2} \varphi+\left(4 a_{5} \alpha^{5}+\left(24 b_{9}-10 a_{8}\right) \alpha^{4}\right. \\
& +4 a_{5} \beta \alpha^{3}+3 \beta\left(a_{8}+12 b_{9}\right) \alpha^{2}-18 a_{5} \beta^{2} \alpha+6 \beta^{2}\left(a_{8}\right. \\
& \left.\left.\left.+b_{9}\right)\right) \sin ^{4} \varphi \cos \varphi-a_{9}\left(10 \alpha^{4}-3 \beta \alpha^{2}-6 \beta^{2}\right) \sin ^{5} \varphi\right) \text {, } \\
& A_{8}=4 \cos ^{4} \varphi \sqrt{\left(\alpha^{2}+2 \beta\right) R^{2} \cos ^{2} \varphi+1}\left(\left(-4 a_{6} \alpha^{5}+8 a_{3} \beta \alpha^{4}-4 \beta\left(5 a_{6}\right.\right.\right. \\
& \left.\left.+3 a_{1} \beta\right) \alpha^{3}+24 a_{3} \beta^{2} \alpha^{2}-3 \beta^{2}\left(5 a_{6}+8 a_{1} \beta\right) \alpha+8 a_{3} \beta^{3}\right) \cos ^{5} \varphi \\
& -\left(4\left(a_{7}+2 b_{6}\right) \alpha^{5}-8 \beta\left(a_{4}+b_{3}\right) \alpha^{4}+4 \beta\left(5 a_{7}+3 a_{2} \beta+2 \beta b_{1}\right.\right. \\
& \left.+4 b_{6}\right) \alpha^{3}-12 \beta^{2}\left(2 a_{4}+b_{3}\right) \alpha^{2}+\beta^{2}\left(15 a_{7}+24 a_{2} \beta+8 \beta b_{1}+6 b_{6}\right) \alpha \\
& \left.-2 \beta^{3}\left(4 a_{4}+b_{3}\right)\right) \sin \varphi \cos ^{4} \varphi+\left(4\left(a_{6}-a_{8}-2 b_{7}\right) \alpha^{5}+8 \beta\left(a_{5}\right.\right. \\
& \left.+b_{4}\right) \alpha^{4}-4 \beta\left(a_{6}+5 a_{8}+a_{1} \beta+2 \beta b_{2}+4 b_{7}\right) \alpha^{3}+12 \beta^{2}\left(a_{3}+2 a_{5}\right. \\
& \left.+b_{4}\right) \alpha^{2}-\beta^{2}\left(9 a_{6}+15 a_{8}+16 a_{1} \beta+8 \beta b_{2}+6 b_{7}\right) \alpha+2 \beta^{3}\left(3 a_{3}\right. \\
& \left.\left.+4 a_{5}+b_{4}\right)\right) \sin ^{2} \varphi \cos ^{3} \varphi+\left(4\left(a_{7}-a_{9}-2 b_{8}\right) \alpha^{5}+8 \beta b_{5} \alpha^{4}\right. \\
& -4 \beta\left(a_{7}+5 a_{9}+a_{2} \beta+4 b_{8}\right) \alpha^{3}+12 \beta^{2}\left(a_{4}+b_{5}\right) \alpha^{2}-\beta^{2}\left(9 a_{7}\right. \\
& \left.\left.+15 a_{9}+16 a_{2} \beta+6 b_{8}\right) \alpha+2 \beta^{3}\left(3 a_{4}+b_{5}\right)\right) \sin ^{3} \varphi \cos ^{2} \varphi+\left(4 \left(a_{8}\right.\right. \\
& \left.-2 b_{9}\right) \alpha^{5}-4 \beta\left(a_{8}+4 b_{9}\right) \alpha^{3}+12 a_{5} \beta^{2} \alpha^{2}-3 \beta^{2}\left(3 a_{8}+2 b_{9}\right) \alpha \\
& \left.\left.+6 a_{5} \beta^{3}\right) \sin ^{4} \varphi \cos \varphi+\alpha a_{9}\left(4 \alpha^{4}-4 \beta \alpha^{2}-9 \beta^{2}\right) \sin ^{5} \varphi\right) \text {, } \\
& A_{9}=4 \cos ^{5} \varphi\left(\left(4 a_{6} \alpha^{6}-8 a_{3} \beta \alpha^{5}+4 \beta\left(7 a_{6}+3 a_{1} \beta\right) \alpha^{4}-36 a_{3} \beta^{2} \alpha^{3}\right.\right. \\
& \left.+\beta^{2}\left(39 a_{6}+40 a_{1} \beta\right) \alpha^{2}-32 a_{3} \beta^{3} \alpha+4 \beta^{3}\left(2 a_{6}+5 a_{1} \beta\right)\right) \cos ^{5} \varphi \\
& +\left(4\left(a_{7}+2 b_{6}\right) \alpha^{6}-8 \beta\left(a_{4}+b_{3}\right) \alpha^{5}+4 \beta\left(7 a_{7}+3 a_{2} \beta+2 \beta b_{1}\right.\right. \\
& \left.+6 b_{6}\right) \alpha^{4}-4 \beta^{2}\left(9 a_{4}+5 b_{3}\right) \alpha^{3}+\beta^{2}\left(39 a_{7}+40 a_{2} \beta+16 \beta b_{1}+18 b_{6}\right) \alpha^{2} \\
& \left.-2 \beta^{3}\left(16 a_{4}+5 b_{3}\right) \alpha+2 \beta^{3}\left(4 a_{7}+10 \alpha_{2} \beta+2 \beta b_{1}+b_{6}\right)\right) \sin \varphi \cos ^{4} \varphi \\
& -\left(4\left(a_{6}-a_{8}-2 b_{7}\right) \alpha^{6}+8 \beta\left(a_{5}+b_{4}\right) \alpha^{5}-4 \beta\left(a_{6}+7 a_{8}+a_{1} \beta+2 \beta b_{2}\right.\right. \\
& \left.+6 b_{7}\right) \alpha^{4}+4 \beta^{2}\left(4 a_{3}+9 a_{5}+5 b_{4}\right) \alpha^{3}-\beta^{2}\left(21 a_{6}+39 a_{8}+24 a_{1} \beta\right. \\
& \left.+16 \beta b_{2}+18 b_{7}\right) \alpha^{2}+2 \beta^{3}\left(11 a_{3}+16 a_{5}+5 b_{4}\right) \alpha-2 \beta^{3}\left(3 a_{6}+4 a_{8}\right. \\
& \left.\left.+8 a_{1} \beta+2 \beta b_{2}+b_{7}\right)\right) \sin ^{2} \varphi \cos ^{3} \varphi-\left(4\left(a_{7}-a_{9}-2 b_{8}\right) \alpha^{6}+8 \beta b_{5} \alpha^{5}\right. \\
& -4 \beta\left(a_{7}+7 a_{9}+a_{2} \beta+6 b_{8}\right) \alpha^{4}+4 \beta^{2}\left(4 a_{4}+5 b_{5}\right) \alpha^{3}-3 \beta^{2}\left(7 a_{7}+13 a_{9}\right. \\
& \left.+8 a_{2} \beta+6 b_{8}\right) \alpha^{2}+2 \beta^{3}\left(11 a_{4}+5 b_{5}\right) \alpha-2 \beta^{3}\left(3 a_{7}+4 a_{9}+8 a_{2} \beta\right. \\
& \left.\left.+b_{8}\right)\right) \sin ^{3} \varphi \cos ^{2} \varphi-\left(4\left(a_{8}-2 b_{9}\right) \alpha^{6}-4 \beta\left(a_{8}+6 b_{9}\right) \alpha^{4}+16 a_{5} \beta^{2} \alpha^{3}\right. \\
& \left.-3 \beta^{2}\left(7 a_{8}+6 b_{9}\right) \alpha^{2}+22 a_{5} \beta^{3} \alpha-2 \beta^{3}\left(3 a_{8}+b_{9}\right)\right) \sin ^{4} \varphi \cos \varphi \\
& \left.-a_{9}\left(4 \alpha^{6}-4 \beta \alpha^{4}-21 \beta^{2} \alpha^{2}-6 \beta^{3}\right) \sin ^{5} \varphi\right) \text {, } \\
& A_{10}=8 \beta \cos ^{6} \varphi \sqrt{\left(\alpha^{2}+2 \beta\right) R^{2} \cos ^{2} \varphi+1}\left(\left(-4 a_{6} \alpha^{5}+4 a_{3} \beta \alpha^{4}-2 \beta\left(5 a_{6}\right.\right.\right.
\end{aligned}
$$




$$
\begin{aligned}
& \left.\left.+2 a_{1} \beta\right) \alpha^{3}+8 a_{3} \beta^{2} \alpha^{2}-\beta^{2}\left(5 a_{6}+6 a_{1} \beta\right) \alpha+2 a_{3} \beta^{3}\right) \cos ^{3} \varphi-\left(4 a_{7} \alpha^{5}\right. \\
& -4 a_{4} \beta \alpha^{4}+2 \beta\left(5 a_{7}+2 a_{2} \beta\right) \alpha^{3}-8 a_{4} \beta^{2} \alpha^{2}+\beta^{2}\left(5 a_{7}+6 a_{2} \beta\right) \alpha \\
& \left.-2 a_{4} \beta^{3}\right) \sin \varphi \cos ^{2} \varphi-\left(4 a_{8} \alpha^{5}-4 a_{5} \beta \alpha^{4}+10 a_{8} \beta \alpha^{3}-8 a_{5} \beta^{2} \alpha^{2}\right. \\
& \left.\left.+5 a_{8} \beta^{2} \alpha-2 a_{5} \beta^{3}\right) \sin ^{2} \varphi \cos \varphi-\alpha a_{9}\left(4 \alpha^{4}+10 \beta \alpha^{2}+5 \beta^{2}\right) \sin ^{3} \varphi\right), \\
A_{11}= & 8 \beta\left(\alpha^{2}+2 \beta\right) \cos ^{7} \varphi\left(\left(4 a_{6} \alpha^{4}-4 a_{3} \beta \alpha^{3}+2 \beta\left(3 a_{6}+2 a_{1} \beta\right) \alpha^{2}\right.\right. \\
& \left.-4 a_{3} \beta^{2} \alpha+\beta^{2}\left(a_{6}+2 a_{1} \beta\right)\right) \cos ^{3} \varphi+\left(4 a_{7} \alpha^{4}-4 a_{4} \beta \alpha^{3}+2 \beta\left(3 a_{7}\right.\right. \\
& \left.\left.+2 a_{2} \beta\right) \alpha^{2}-4 a_{4} \beta^{2} \alpha+\beta^{2}\left(a_{7}+2 a_{2} \beta\right)\right) \sin \varphi \cos ^{2} \varphi+\left(4 a_{8} \alpha^{4}\right. \\
& \left.-4 a_{5} \beta \alpha^{3}+6 a_{8} \beta \alpha^{2}-4 a_{5} \beta^{2} \alpha+a_{8} \beta^{2}\right) \sin ^{2} \varphi \cos \varphi \\
& \left.+a_{9}\left(4 \alpha^{4}+6 \beta \alpha^{2}+\beta^{2}\right) \sin ^{3} \varphi\right) \\
B= & \left.-2 \beta R^{2} \cos ^{2} \varphi-1\right)\left(2\left(\alpha^{2}+\beta\right) R^{2} \cos ^{2} \varphi\right. \\
& \left.-2 \alpha R \sqrt{\left(\alpha^{2}+2 \beta\right) R^{2} \cos ^{2} \varphi+1} \cos \varphi+1\right)^{2}\left(\left(\alpha^{2}+2 \beta\right) R^{2} \cos ^{2} \varphi\right. \\
& \left.-\alpha R \sqrt{\left(\alpha^{2}+2 \beta\right) R^{2} \cos ^{2} \varphi+1} \cos \varphi+1\right) .
\end{aligned}
$$

For proving that $B$ is non-zero we have to show that equations

$$
\begin{aligned}
2\left(\alpha^{2}+\beta\right) R^{2} \cos ^{2} \varphi & =2 \alpha R \sqrt{\left(\alpha^{2}+2 \beta\right) R^{2} \cos ^{2} \varphi+1} \cos \varphi+1, \\
\left(\alpha^{2}+2 \beta\right) R^{2} \cos ^{2} \varphi & =\alpha R \sqrt{\left(\alpha^{2}+2 \beta\right) R^{2} \cos ^{2} \varphi+1} \cos \varphi+1,
\end{aligned}
$$

do not have solutions in the interval $(0, \sqrt{-1 /(2 \beta)})$. In fact we take the square in both sides of the two previous equations and we obtain respectively

$$
\left(2 \beta R^{2} \cos ^{2} \varphi+1\right)^{2}=0, \quad\left(2 \beta R^{2} \cos ^{2} \varphi+1\right)\left(R^{2}\left(\alpha^{2}+2 \beta\right) \cos ^{2} \varphi+1\right)=0 .
$$

Since $R \in(0, \sqrt{-1 /(2 \beta)})$ the previous two equations do not have solutions. So $x Q(x, y)-y P(x, y) \neq 0$ in the period annulus of the unperturbed center

Now we compute the averaged function $f:(0, \sqrt{-1 / 2 \beta}) \rightarrow \mathbb{R}$ defined by

$$
f(R)=\int_{0}^{2 \pi} \sum_{i=1}^{11} \frac{A_{i}(\varphi, \alpha, \beta, a, b)}{B(R, \varphi, \alpha, \beta)} R^{i} d \varphi .
$$

By computing the previous integral, we obtain that $f(R)=N(R) / D(R)$, where

$$
\begin{aligned}
N(R)= & \beta\left(a_{6}+3 a_{8}+2 a_{1} \beta+2 \beta b_{2}+b_{7}-3 b_{9}\right) g_{0}-2 \beta^{2}\left(2 a_{1} \alpha^{2}+a_{3} \alpha\right. \\
& \left.+a_{5} \alpha+a_{6}+a_{8}+2 a_{1} \beta\right) g_{1}+2 \beta\left(a_{8}+b_{9}\right) g_{2}+\left(a_{6}-a_{8}-b_{7}\right. \\
& \left.+b_{9}\right) g_{3}, \\
D(R)= & 4 \beta^{2} R, \quad g_{0}=R^{2}, \quad g_{1}=R^{4}, \quad g_{2}=R^{2} \sqrt{1+2 \beta R^{2}}, \\
g_{3}= & 1-\sqrt{1+2 \beta R^{2}} .
\end{aligned}
$$

The previous computations were verified using the software Mathematica. The zeros of the function $f$ correspond to zeros of the function $N$. In order to 
find the maximum number of zeros of $f$, we have to prove that $\left(g_{0}, g_{1}, g_{2}, g_{3}\right)$ is an ECT-system, and this is the case if $W\left(g_{0}, \ldots, g_{k}\right)(R) \neq 0$, for $0 \leq k \leq 3$, where $W\left(g_{0}, \ldots, g_{k}\right)(R)$ denotes the Wronskians of the functions $\left(g_{0}, \ldots, g_{k}\right)$. More precisely we have

$$
\begin{aligned}
& W\left(g_{0}\right)(R)=R^{2}, \quad W\left(g_{0}, g_{1}\right)=2 R^{5}, \quad W\left(g_{0}, g_{1}, g_{2}\right)(R)=-\frac{8 R^{9} \beta^{2}}{\left(1+2 \beta R^{2}\right)^{3 / 2}}, \\
& \begin{aligned}
W\left(g_{0}, \ldots, g_{3}\right)(R)= & \frac{-192 \beta^{2} R^{6}}{\left(1+2 \beta R^{2}\right)^{7 / 2}}\left[\sqrt{1+2 \beta R^{2}} R^{4} \beta^{2}+4 \sqrt{1+2 \beta R^{2}} \beta R^{2}\right. \\
& \left.+2 \sqrt{1+2 \beta R^{2}}-4 R^{4} \beta^{2}-6 \beta R^{2}-2\right] .
\end{aligned}
\end{aligned}
$$

Since $\beta<0$ and $R \in(0, \sqrt{-1 /(2 \beta)})$, the first three Wronskians are nonzero. Now, to get the zeros of $W\left(g_{0}, \ldots g_{3}\right)(R)$ is equivalent to solve the following equation

$$
\sqrt{1+2 \beta R^{2}} R^{4} \beta^{2}+4 \sqrt{1+2 \beta R^{2}} \beta R^{2}+2 \sqrt{1+2 \beta R^{2}}=4 R^{4} \beta^{2}+6 \beta R^{2}+2 .
$$

We take the square in both sides of the previous equation and after some simplifications we get

$$
\left(1+2 \beta R^{2}\right) R^{8} \beta^{4}=0,
$$

which is it impossible because $R \in(0, \sqrt{-1 /(2 \beta)}$. Thus, the Wronskian $W\left(g_{0}, \ldots g_{3}\right)(R)$ is non-zero in $(0, \sqrt{-1 /(2 \beta)})$. Hence, since $\left(g_{0}, g_{1}, g_{2}, g_{3}\right)$ is an ECT-system $f$ has at most 3 zeros and they are reached.

System (11) is analytic and satisfies the assumptions of Theorem 3. Therefore the zeros of $f$ correspond to periodic orbits of perturbed system (2) and Theorem 1 follows.

\section{Proof of Theorem 2}

The functions $H, \mu$ and $\rho$ are the same than the ones given in the proof of Theorem 1. Denote $a=\left(a_{1}, \ldots, a_{9}\right), b=\left(b_{1}, \ldots, b_{9}\right), c=\left(c_{1}, \ldots, c_{9}\right)$, $d=\left(d_{1}, \ldots, d_{9}\right), j_{1}=(a, b, \alpha, \beta)$ and $j_{2}=(c, d, \alpha, \beta)$. We transform system (3) into the system

$$
\frac{d R}{d \varphi}=\left\{\begin{array}{cc}
\varepsilon \frac{\mu\left(x^{2}+y^{2}\right)\left(Q p_{1}-P q_{1}\right)}{2 R(Q x-P y)}+\mathcal{O}\left(\varepsilon^{2}\right) & \text { if } \quad y>0, \\
\varepsilon \frac{\mu\left(x^{2}+y^{2}\right)\left(Q p_{2}-P q_{2}\right)}{2 R(Q x-P y)}+\mathcal{O}\left(\varepsilon^{2}\right) & \text { if } \quad y<0 .
\end{array}\right.
$$

As in the proof of Theorem 1, we have $R(Q x-P y) \neq 0$. We must study the zeros of the averaged function $f:(0, \sqrt{-1 /(2 \beta)}) \rightarrow \mathbb{R}$ given by

$$
f(R)=\int_{0}^{\pi} \frac{\mu\left(x^{2}+y^{2}\right)\left(Q p_{1}-P q_{1}\right)}{2 R(Q x-P y)} d \varphi+\int_{\pi}^{2 \pi} \frac{\mu\left(x^{2}+y^{2}\right)\left(Q p_{2}-P q_{2}\right)}{2 R(Q x-P y)} d \varphi .
$$


Computing these integrals we get

$$
f(R)=\sum_{i=0}^{21} f_{i} B_{i}
$$

where

$$
\begin{aligned}
f_{0}= & \frac{1}{R}\left(\sqrt{1+2 \beta R^{2}}-1\right), \quad f_{1}=R \sqrt{1+2 \beta R^{2}}, \quad f_{2}=R, \quad f_{3}=R^{3}, \\
f_{i}= & \left\{\begin{array}{l}
\frac{\sqrt{1+\left(\alpha^{2}+2 \beta\right) R^{2}}}{1+2 \beta R^{2}} R^{2 i-8}, \quad \text { for } i=4, \ldots, 8, \\
\frac{1}{2 R}\left(1+2 \beta R^{2}\right) \log \left(\frac{a R+\sqrt{1+\left(\alpha^{2}+2 \beta\right) R^{2}}}{a R-\sqrt{1+\left(\alpha^{2}+2 \beta\right) R^{2}}}\right)^{2}, \quad \text { for } i=9, \\
\frac{1}{2} R^{2 i-19}\left(1+2 \beta R^{2}\right) \log \left(\frac{a R+\sqrt{1+\left(\alpha^{2}+2 \beta\right) R^{2}}}{a R-\sqrt{1+\left(\alpha^{2}+2 \beta\right) R^{2}}}\right)^{2}, \\
\frac{1}{R} \sinh ^{-1}\left(R \sqrt{\alpha^{2}+2 \beta}\right), \quad \text { for } i=14, \\
R \sinh ^{-1}\left(R \sqrt{\alpha^{2}+2 \beta}\right), \quad \text { for } i=15, \\
\frac{1}{R} \tanh ^{-1}\left(\frac{a R}{\sqrt{1+\left(\alpha^{2}+2 \beta\right) R^{2}}}\right), \quad \text { for } i=16, \\
R^{2 i-33} \tanh ^{-1}\left(\frac{a R}{\sqrt{1+\left(\alpha^{2}+2 \beta\right) R^{2}}}\right), \quad \text { for } i=17, \ldots, 21
\end{array}\right.
\end{aligned}
$$

and

$$
\begin{array}{ccll}
B_{0}=C_{0}\left(-P_{0}\left(j_{1}\right)+P_{0}\left(j_{2}\right)\right), & B_{1}=C_{1}\left(P_{1}\left(j_{1}\right)-P_{1}\left(j_{2}\right)\right), \\
B_{2}=C_{2}\left(-P_{2}\left(j_{1}\right)-P_{2}\left(j_{2}\right)\right), & B_{3}=C_{3}\left(-P_{3}\left(j_{1}\right)-P_{3}\left(j_{2}\right)\right), \\
B_{4}=C_{4}\left(P_{4}\left(j_{1}\right)-P_{4}\left(j_{2}\right)\right), & B_{5}=C_{5}\left(-P_{5}\left(j_{1}\right)+P_{5}\left(j_{2}\right)\right), \\
B_{6}=C_{6}\left(-P_{6}\left(j_{1}\right)+P_{6}\left(j_{2}\right)\right), & B_{7}=C_{7}\left(P_{7}\left(j_{1}\right)-P_{7}\left(j_{2}\right)\right), \\
B_{8}=C_{8}\left(-P_{8}\left(j_{1}\right)+P_{8}\left(j_{2}\right)\right), & B_{9}=C_{9}\left(P_{9}\left(j_{1}\right)-P_{9}\left(j_{2}\right)\right), \\
B_{10}=C_{10}\left(-P_{10}\left(j_{1}\right)+P_{10}\left(j_{2}\right)\right), & B_{11}=C_{11}\left(P_{11}\left(j_{1}\right)-P_{11}\left(j_{2}\right)\right), \\
B_{12}=C_{12}\left(-P_{12}\left(j_{1}\right)+P_{12}\left(j_{2}\right)\right), & B_{13}=C_{13}\left(P_{13}\left(j_{1}\right)-P_{13}\left(j_{2}\right)\right), \\
B_{14}=C_{14}\left(-P_{14}\left(j_{1}\right)+P_{14}\left(j_{2}\right)\right), & B_{15}=C_{15}\left(-P_{15}\left(j_{1}\right)+P_{15}\left(j_{2}\right)\right), \\
B_{16}=C_{16}\left(-P_{16}\left(j_{1}\right)+P_{16}\left(j_{2}\right)\right), & B_{17}=C_{17}\left(P_{17}\left(j_{1}\right)-P_{17}\left(j_{2}\right)\right), \\
B_{18}=C_{18}\left(P_{18}\left(j_{1}\right)-P_{18}\left(j_{2}\right)\right), & B_{19}=C_{19}\left(-P_{19}\left(j_{1}\right)+P_{19}\left(j_{2}\right)\right), \\
B_{20}=C_{20}\left(P_{20}\left(j_{1}\right)-P_{20}\left(j_{2}\right)\right), & B_{21}=C_{21}\left(-P_{21}\left(j_{1}\right)+P_{21}\left(j_{2}\right)\right),
\end{array}
$$

with 


$$
\begin{aligned}
& C_{0}=\frac{\pi}{4 \beta^{2}}, \quad C_{1}=\quad \frac{\pi}{2 \beta}, \quad C_{2}=\frac{\pi}{4 \beta}, \\
& C_{3}=\frac{\pi}{2}, \quad C_{4}=\frac{1}{2 \alpha^{5} \beta\left(\alpha^{2}+2 \beta\right)}, \quad C_{5}=\frac{1}{\alpha^{5}\left(\alpha^{2}+2 \beta\right)}, \\
& C_{6}=\frac{2}{\alpha^{5}}, \quad C_{7}=\frac{8(\beta-1) \beta}{\alpha^{5}}, \quad C_{8}=2 C_{7}\left(\alpha^{2}+2 \beta\right) \text {, } \\
& C_{9,10}=\frac{1}{4 \alpha^{8} \beta^{6}}, \quad C_{11}=\frac{\alpha^{2}+2 \beta}{2 \alpha^{8} \beta^{5}}, \quad C_{12}=\frac{4\left(\alpha^{2}+2 \beta\right)^{2}}{\alpha^{8}}, \\
& C_{13,21}=\frac{1}{\alpha^{8}}, \quad C_{14}=\frac{1}{2 \beta^{2}\left(\alpha^{2}+2 \beta\right)^{3 / 2}}, \quad C_{15}=\frac{1}{\beta \sqrt{\alpha^{2}+2 \beta}}, \\
& C_{16,17}=\frac{1}{2 \alpha^{8} \beta^{6}}, \quad C_{18}=\quad \frac{1}{\alpha^{8} \beta^{5}}, \quad C_{19}=\frac{2}{\alpha^{8} \beta^{4}}, \\
& C_{20}=\frac{16 \beta\left(\alpha^{2}+2 \beta\right)}{\alpha^{8}}, \\
& P_{0}=c_{6}-c_{8}-d_{7}+d_{9} \\
& P_{1}=c_{8}-d_{9} \\
& P_{2}=2 \beta c_{1}+2 \beta d_{2}-c_{6}+3 c_{8}+d_{7}-3 d_{9}, \\
& P_{3}=2 \alpha^{2} c_{1}+\alpha c_{3}+\alpha c_{5}+2 \beta c_{1}+c_{6}+c_{8}, \\
& P_{4}=-2 \alpha^{6} \beta c_{2}-2 \alpha^{6} \beta d_{1}+\alpha^{6} c_{7}-\alpha^{6} c_{9}+\alpha^{6} d_{6}-\alpha^{6} d_{8}-2 \alpha^{5} \beta c_{4} \\
& -2 \alpha^{5} \beta d_{3}+2 \alpha^{5} \beta d_{5}-32 \alpha^{4} \beta^{2} c_{2}-32 \alpha^{4} \beta^{2} d_{1}+32 \alpha^{4} \beta c_{2}+32 \alpha^{4} \beta d_{1} \\
& +20 \alpha^{3} \beta^{2} c_{4}+20 \alpha^{3} \beta^{2} d_{3}-20 \alpha^{3} \beta^{2} d_{5}-20 \alpha^{3} \beta c_{4}-20 \alpha^{3} \beta d_{3} \\
& +20 \alpha^{3} \beta d_{5}-96 \alpha^{2} \beta^{3} c_{2}-96 \alpha^{2} \beta^{3} d_{1}+96 \alpha^{2} \beta^{2} c_{2}-4 \alpha^{2} \beta^{2} c_{7} \\
& +4 \alpha^{2} \beta^{2} c_{9}+96 \alpha^{2} \beta^{2} d_{1}-4 \alpha^{2} \beta^{2} d_{6}+4 \alpha^{2} \beta^{2} d_{8}+4 \alpha^{2} \beta c_{7}-4 \alpha^{2} \beta c_{9} \\
& +4 \alpha^{2} \beta d_{6}-4 \alpha^{2} \beta d_{8}+40 \alpha \beta^{3} c_{4}+40 \alpha \beta^{3} d_{3}-40 \alpha \beta^{3} d_{5}-40 \alpha \beta^{2} c_{4} \\
& -40 \alpha \beta^{2} d_{3}+40 \alpha \beta^{2} d_{5}-64 \beta^{4} c_{2}-64 \beta^{4} d_{1}+64 \beta^{3} c_{2}-8 \beta^{3} c_{7} \\
& +8 \beta^{3} c_{9}+64 \beta^{3} d_{1}-8 \beta^{3} d_{6}+8 \beta^{3} d_{8}+8 \beta^{2} c_{7}-8 \beta^{2} c_{9}+8 \beta^{2} d_{6} \\
& -8 \beta^{2} d_{8} \\
& P_{5}=2 \alpha^{8} c_{2}+\alpha^{7} c_{4}+14 \alpha^{6} \beta c_{2}+18 \alpha^{6} \beta d_{1}-8 \alpha^{6} c_{2}-\alpha^{6} c_{7}+\alpha^{6} c_{9} \\
& -16 \alpha^{6} d_{1}-\alpha^{6} d_{6}+\alpha^{6} d_{8}-20 \alpha^{5} \beta c_{4}-38 \alpha^{5} \beta d_{3}-2 \alpha^{5} \beta d_{5}+24 \alpha^{5} c_{4} \\
& +40 \alpha^{5} d_{3}+96 \alpha^{4} \beta^{2} c_{2}+128 \alpha^{4} \beta^{2} d_{1}-96 \alpha^{4} \beta c_{2}+26 \alpha^{4} \beta c_{7} \\
& +10 \alpha^{4} \beta c_{9}-128 \alpha^{4} \beta d_{1}+36 \alpha^{4} \beta d_{6}-26 \alpha^{4} c_{7}-10 \alpha^{4} c_{9}-36 \alpha^{4} d_{6} \\
& -112 \alpha^{3} \beta^{2} c_{4}-140 \alpha^{3} \beta^{2} d_{3}+112 \alpha^{3} \beta c_{4}+140 \alpha^{3} \beta d_{3}+240 \alpha^{2} \beta^{3} c_{2} \\
& +240 \alpha^{2} \beta^{3} d_{1}-240 \alpha^{2} \beta^{2} c_{2}+68 \alpha^{2} \beta^{2} c_{7}+16 \alpha^{2} \beta^{2} c_{9}-240 \alpha^{2} \beta^{2} d_{1} \\
& +84 \alpha^{2} \beta^{2} d_{6}-68 \alpha^{2} \beta c_{7}-16 \alpha^{2} \beta c_{9}-84 \alpha^{2} \beta d_{6}-128 \alpha \beta^{3} c_{4} \\
& -120 \alpha \beta^{3} d_{3}+128 \alpha \beta^{2} c_{4}+120 \alpha \beta^{2} d_{3}+160 \beta^{4} c_{2}+96 \beta^{4} d_{1}
\end{aligned}
$$




$$
\begin{aligned}
& -160 \beta^{3} c_{2}+32 \beta^{3} c_{7}-8 \beta^{3} c_{9}-96 \beta^{3} d_{1}+24 \beta^{3} d_{6}-32 \beta^{2} c_{7}+8 \beta^{2} c_{9} \\
& -24 \beta^{2} d_{6} \text {, } \\
& P_{6}=2 \alpha^{6} \beta c_{2}-7 \alpha^{5} \beta c_{4}-16 \alpha^{5} \beta d_{3}+8 \alpha^{5} c_{4}+16 \alpha^{5} d_{3}+24 \alpha^{4} \beta^{2} c_{2} \\
& +32 \alpha^{4} \beta^{2} d_{1}-24 \alpha^{4} \beta c_{2}+28 \alpha^{4} \beta c_{7}-32 \alpha^{4} \beta d_{1}+48 \alpha^{4} \beta d_{6}-28 \alpha^{4} c_{7} \\
& -48 \alpha^{4} d_{6}-72 \alpha^{3} \beta^{2} c_{4}-80 \alpha^{3} \beta^{2} d_{3}+72 \alpha^{3} \beta c_{4}+80 \alpha^{3} \beta d_{3} \\
& +120 \alpha^{2} \beta^{3} c_{2}+96 \alpha^{2} \beta^{3} d_{1}-120 \alpha^{2} \beta^{2} c_{2}+78 \alpha^{2} \beta^{2} c_{7}-96 \alpha^{2} \beta^{2} d_{1} \\
& +72 \alpha^{2} \beta^{2} d_{6}-78 \alpha^{2} \beta c_{7}-72 \alpha^{2} \beta d_{6}-96 \alpha \beta^{3} c_{4}-60 \alpha \beta^{3} d_{3} \\
& +96 \alpha \beta^{2} c_{4}+60 \alpha \beta^{2} d_{3}+80 \beta^{4} c_{2}+32 \beta^{4} d_{1}-80 \beta^{3} c_{2}+24 \beta^{3} c_{7} \\
& -32 \beta^{3} d_{1}+12 \beta^{3} d_{6}-24 \beta^{2} c_{7}-12 \beta^{2} d_{6} \text {, } \\
& P_{7}=4 \alpha^{6} c_{7}-8 \alpha^{6} c_{9}-8 \alpha^{6} d_{8}+8 \alpha^{5} \beta d_{5}-4 \alpha^{4} \beta^{2} c_{2}-4 \alpha^{4} \beta c_{7}-24 \alpha^{4} \beta c_{9} \\
& -24 \alpha^{4} \beta d_{8}+16 \alpha^{3} \beta^{2} c_{4}+20 \alpha^{3} \beta^{2} d_{5}-24 \alpha^{2} \beta^{3} c_{2}-21 \alpha^{2} \beta^{2} c_{7} \\
& -18 \alpha^{2} \beta^{2} c_{9}-18 \alpha^{2} \beta^{2} d_{8}+22 \alpha \beta^{3} c_{4}+10 \alpha \beta^{3} d_{5}-16 \beta^{4} c_{2}-6 \beta^{3} c_{7} \\
& -2 \beta^{3} c_{9}-2 \beta^{3} d_{8} \text {, } \\
& P_{8}=32(\beta-1) \beta c_{9}\left(\alpha^{2}+2 \beta\right)\left(4 \alpha^{4}+6 \alpha^{2} \beta+\beta^{2}\right) \text {, } \\
& P_{9}=-16 \alpha^{17} c_{4}-16 \alpha^{17} d_{3}+16 \alpha^{17} d_{5}+32 \alpha^{16} \beta c_{2}+32 \alpha^{16} \beta d_{1}+48 \alpha^{16} c_{7} \\
& -48 \alpha^{16} c_{9}+48 \alpha^{16} d_{6}-48 \alpha^{16} d_{8}-120 \alpha^{15} \beta c_{4}-120 \alpha^{15} \beta d_{3} \\
& +120 \alpha^{15} \beta d_{5}+176 \alpha^{14} \beta^{2} c_{2}+176 \alpha^{14} \beta^{2} d_{1}+192 \alpha^{14} \beta c_{7}-192 \alpha^{14} \beta c_{9} \\
& +192 \alpha^{14} \beta d_{6}-192 \alpha^{14} \beta d_{8}-280 \alpha^{13} \beta^{2} c_{4}-280 \alpha^{13} \beta^{2} d_{3} \\
& +280 \alpha^{13} \beta^{2} d_{5}+312 \alpha^{12} \beta^{3} c_{2}+312 \alpha^{12} \beta^{3} d_{1}+252 \alpha^{12} \beta^{2} c_{7} \\
& -252 \alpha^{12} \beta^{2} c_{9}+252 \alpha^{12} \beta^{2} d_{6}-252 \alpha^{12} \beta^{2} d_{8}-250 \alpha^{11} \beta^{3} c_{4} \\
& -250 \alpha^{11} \beta^{3} d_{3}+250 \alpha^{11} \beta^{3} d_{5}+200 \alpha^{10} \beta^{4} c_{2}+200 \alpha^{10} \beta^{4} d_{1} \\
& +120 \alpha^{10} \beta^{3} c_{7}-120 \alpha^{10} \beta^{3} c_{9}+120 \alpha^{10} \beta^{3} d_{6}-120 \alpha^{10} \beta^{3} d_{8} \\
& -75 \alpha^{9} \beta^{4} c_{4}-75 \alpha^{9} \beta^{4} d_{3}+75 \alpha^{9} \beta^{4} d_{5}+40 \alpha^{8} \beta^{5} c_{2}+40 \alpha^{8} \beta^{5} d_{1} \\
& +15 \alpha^{8} \beta^{4} c_{7}-15 \alpha^{8} \beta^{4} c_{9}+15 \alpha^{8} \beta^{4} d_{6}-15 \alpha^{8} \beta^{4} d_{8}+16 \alpha^{6} \beta^{6} c_{2} \\
& +16 \alpha^{6} \beta^{6} d_{1}-8 \alpha^{5} \beta^{6} c_{4}-8 \alpha^{5} \beta^{6} d_{3}+8 \alpha^{5} \beta^{6} d_{5}+64 \alpha^{4} \beta^{7} c_{2} \\
& +64 \alpha^{4} \beta^{7} d_{1}-60 \alpha^{4} \beta^{6} c_{7}+60 \alpha^{4} \beta^{6} c_{9}-60 \alpha^{4} \beta^{6} d_{6}+60 \alpha^{4} \beta^{6} d_{8} \\
& +20 \alpha^{3} \beta^{7} c_{4}+20 \alpha^{3} \beta^{7} d_{3}-20 \alpha^{3} \beta^{7} d_{5}+48 \alpha^{2} \beta^{8} c_{2}+48 \alpha^{2} \beta^{8} d_{1} \\
& -60 \alpha^{2} \beta^{7} c_{7}+60 \alpha^{2} \beta^{7} c_{9}-60 \alpha^{2} \beta^{7} d_{6}+60 \alpha^{2} \beta^{7} d_{8}+32 \beta^{9} c_{2}+32 \beta^{9} d_{1} \text {, } \\
& P_{10}=16 \alpha^{18} c_{7}-48 \alpha^{18} c_{9}-32 \alpha^{18} d_{8}+32 \alpha^{17} \beta d_{5}-16 \alpha^{16} \beta^{2} c_{2}+24 \alpha^{16} \beta c_{7} \\
& -200 \alpha^{16} \beta c_{9}-176 \alpha^{16} \beta d_{8}+64 \alpha^{15} \beta^{2} c_{4}+160 \alpha^{15} \beta^{2} d_{5}-136 \alpha^{14} \beta^{3} c_{2} \\
& -104 \alpha^{14} \beta^{2} c_{7}-248 \alpha^{14} \beta^{2} c_{9}-352 \alpha^{14} \beta^{2} d_{8}+248 \alpha^{13} \beta^{3} c_{4} \\
& +280 \alpha^{13} \beta^{3} d_{5}-324 \alpha^{12} \beta^{4} c_{2}-254 \alpha^{12} \beta^{3} c_{7}-54 \alpha^{12} \beta^{3} c_{9} \\
& -308 \alpha^{12} \beta^{3} d_{8}+300 \alpha^{11} \beta^{4} c_{4}+200 \alpha^{11} \beta^{4} d_{5}-280 \alpha^{10} \beta^{5} c_{2} \\
& -165 \alpha^{10} \beta^{4} c_{7}+55 \alpha^{10} \beta^{4} c_{9}-110 \alpha^{10} \beta^{4} d_{8}+110 \alpha^{9} \beta^{5} c_{4}+50 \alpha^{9} \beta^{5} d_{5} \\
& -112 \alpha^{8} \beta^{6} c_{2}-32 \alpha^{8} \beta^{6} d_{1}-30 \alpha^{8} \beta^{5} c_{7}+20 \alpha^{8} \beta^{5} c_{9}-10 \alpha^{8} \beta^{5} d_{8} \\
& +32 \alpha^{7} \beta^{6} c_{4}+48 \alpha^{7} \beta^{6} d_{3}-16 \alpha^{7} \beta^{6} d_{5}-240 \alpha^{6} \beta^{7} c_{2}-256 \alpha^{6} \beta^{7} d_{1}
\end{aligned}
$$




$$
\begin{aligned}
& +32 \alpha^{6} \beta^{6} c_{7}-64 \alpha^{6} \beta^{6} c_{9}+24 \alpha^{6} \beta^{6} d_{6}-56 \alpha^{6} \beta^{6} d_{8}+168 \alpha^{5} \beta^{7} c_{4} \\
& +216 \alpha^{5} \beta^{7} d_{3}-56 \alpha^{5} \beta^{7} d_{5}-656 \alpha^{4} \beta^{8} c_{2}-672 \alpha^{4} \beta^{8} d_{1}+124 \alpha^{4} \beta^{7} c_{7} \\
& -268 \alpha^{4} \beta^{7} c_{9}+24 \alpha^{4} \beta^{7} d_{6}-168 \alpha^{4} \beta^{7} d_{8}+272 \alpha^{3} \beta^{8} c_{4}+360 \alpha^{3} \beta^{8} d_{3} \\
& -80 \alpha^{3} \beta^{8} d_{5}-704 \alpha^{2} \beta^{9} c_{2}-704 \alpha^{2} \beta^{9} d_{1}+48 \alpha^{2} \beta^{8} c_{7}-216 \alpha^{2} \beta^{8} c_{9} \\
& -72 \alpha^{2} \beta^{8} d_{6}-96 \alpha^{2} \beta^{8} d_{8}+208 \alpha \beta^{9} c_{4}+240 \alpha \beta^{9} d_{3}-80 \alpha \beta^{9} d_{5} \\
& -320 \beta^{10} c_{2}-256 \beta^{10} d_{1}-48 \beta^{9} c_{7}+16 \beta^{9} c_{9}-48 \beta^{9} d_{6}+16 \beta^{9} d_{8} \\
& P_{11}=16 \alpha^{16} c_{9}+64 \alpha^{14} \beta c_{9}+84 \alpha^{12} \beta^{2} c_{9}+40 \alpha^{10} \beta^{3} c_{9}+5 \alpha^{8} \beta^{4} c_{9} \\
& -16 \alpha^{7} \beta^{5} c_{4}-32 \alpha^{7} \beta^{5} d_{3}+48 \alpha^{6} \beta^{6} c_{2}+64 \alpha^{6} \beta^{6} d_{1}+72 \alpha^{6} \beta^{5} c_{7} \\
& -24 \alpha^{6} \beta^{5} c_{9}+96 \alpha^{6} \beta^{5} d_{6}-32 \alpha^{6} \beta^{5} d_{8}-176 \alpha^{5} \beta^{6} c_{4}-224 \alpha^{5} \beta^{6} d_{3} \\
& +32 \alpha^{5} \beta^{6} d_{5}+320 \alpha^{4} \beta^{7} c_{2}+320 \alpha^{4} \beta^{7} d_{1}+252 \alpha^{4} \beta^{6} c_{7}-28 \alpha^{4} \beta^{6} c_{9} \\
& +336 \alpha^{4} \beta^{6} d_{6}-96 \alpha^{4} \beta^{6} d_{8}-416 \alpha^{3} \beta^{7} c_{4}-440 \alpha^{3} \beta^{7} d_{3}+80 \alpha^{3} \beta^{7} d_{5} \\
& +544 \alpha^{2} \beta^{8} c_{2}+448 \alpha^{2} \beta^{8} d_{1}+276 \alpha^{2} \beta^{7} c_{7}+312 \alpha^{2} \beta^{7} d_{6}-72 \alpha^{2} \beta^{7} d_{8} \\
& -296 \alpha \beta^{8} c_{4}-240 \alpha \beta^{8} d_{3}+40 \alpha \beta^{8} d_{5}+256 \beta^{9} c_{2}+128 \beta^{9} d_{1} \\
& +72 \beta^{8} c_{7}-16 \beta^{8} c_{9}+48 \beta^{8} d_{6}-8 \beta^{8} d_{8} \text {, } \\
& P_{12}=4 \alpha^{6} c_{7}-8 \alpha^{6} c_{9}-8 \alpha^{6} d_{8}+8 \alpha^{5} \beta d_{5}-4 \alpha^{4} \beta^{2} c_{2}-4 \alpha^{4} \beta c_{7}-20 \alpha^{4} \beta c_{9} \\
& -24 \alpha^{4} \beta d_{8}+16 \alpha^{3} \beta^{2} c_{4}+20 \alpha^{3} \beta^{2} d_{5}-24 \alpha^{2} \beta^{3} c_{2}-21 \alpha^{2} \beta^{2} c_{7} \\
& -12 \alpha^{2} \beta^{2} c_{9}-18 \alpha^{2} \beta^{2} d_{8}+22 \alpha \beta^{3} c_{4}+10 \alpha \beta^{3} d_{5}-16 \beta^{4} c_{2}-6 \beta^{3} c_{7} \\
& -\beta^{3} c_{9}-2 \beta^{3} d_{8} \text {, } \\
& P_{13}=8 \beta c_{9}\left(\alpha^{2}+2 \beta\right)^{3}\left(4 \alpha^{4}+6 \alpha^{2} \beta+\beta^{2}\right) \text {, } \\
& P_{14}=\alpha^{3} c_{7}-\alpha^{3} c_{9}+\alpha^{3} d_{6}-\alpha^{3} d_{8}-2 \alpha \beta^{2} c_{2}-2 \alpha \beta^{2} d_{1}+3 \alpha \beta c_{7}-3 \alpha \beta c_{9} \\
& +3 \alpha \beta d_{6}-3 \alpha \beta d_{8}-2 \beta^{2} c_{4}-2 \beta^{2} d_{3}+2 \beta^{2} d_{5} \text {, } \\
& P_{15}=\alpha c_{7}-2 \alpha c_{9}-\alpha d_{8}-\beta c_{4}+2 \beta d_{5} \text {, } \\
& P_{16}=-16 \alpha^{17} c_{4}-16 \alpha^{17} d_{3}+16 \alpha^{17} d_{5}+32 \alpha^{16} \beta c_{2}+32 \alpha^{16} \beta d_{1}+48 \alpha^{16} c_{7} \\
& -48 \alpha^{16} c_{9}+48 \alpha^{16} d_{6}-48 \alpha^{16} d_{8}-120 \alpha^{15} \beta c_{4}-120 \alpha^{15} \beta d_{3} \\
& +120 \alpha^{15} \beta d_{5}+176 \alpha^{14} \beta^{2} c_{2}+176 \alpha^{14} \beta^{2} d_{1}+192 \alpha^{14} \beta c_{7}-192 \alpha^{14} \beta c_{9} \\
& +192 \alpha^{14} \beta d_{6}-192 \alpha^{14} \beta d_{8}-280 \alpha^{13} \beta^{2} c_{4}-280 \alpha^{13} \beta^{2} d_{3}+280 \alpha^{13} \beta^{2} d_{5} \\
& +312 \alpha^{12} \beta^{3} c_{2}+312 \alpha^{12} \beta^{3} d_{1}+252 \alpha^{12} \beta^{2} c_{7}-252 \alpha^{12} \beta^{2} c_{9} \\
& +252 \alpha^{12} \beta^{2} d_{6}-252 \alpha^{12} \beta^{2} d_{8}-250 \alpha^{11} \beta^{3} c_{4}-250 \alpha^{11} \beta^{3} d_{3} \\
& +250 \alpha^{11} \beta^{3} d_{5}+200 \alpha^{10} \beta^{4} c_{2}+200 \alpha^{10} \beta^{4} d_{1}+120 \alpha^{10} \beta^{3} c_{7} \\
& -120 \alpha^{10} \beta^{3} c_{9}+120 \alpha^{10} \beta^{3} d_{6}-120 \alpha^{10} \beta^{3} d_{8}-75 \alpha^{9} \beta^{4} c_{4}-75 \alpha^{9} \beta^{4} d_{3} \\
& +75 \alpha^{9} \beta^{4} d_{5}+40 \alpha^{8} \beta^{5} c_{2}+40 \alpha^{8} \beta^{5} d_{1}+14 \alpha^{8} \beta^{4} c_{7}-14 \alpha^{8} \beta^{4} c_{9} \\
& +14 \alpha^{8} \beta^{4} d_{6}-14 \alpha^{8} \beta^{4} d_{8}+16 \alpha^{6} \beta^{6} c_{2}+16 \alpha^{6} \beta^{6} d_{1}-8 \alpha^{5} \beta^{6} c_{4} \\
& -8 \alpha^{5} \beta^{6} d_{3}+8 \alpha^{5} \beta^{6} d_{5}+96 \alpha^{4} \beta^{7} c_{2}+96 \alpha^{4} \beta^{7} d_{1}-32 \alpha^{4} \beta^{6} c_{2} \\
& -60 \alpha^{4} \beta^{6} c_{7}+60 \alpha^{4} \beta^{6} c_{9}-32 \alpha^{4} \beta^{6} d_{1}-60 \alpha^{4} \beta^{6} d_{6}+60 \alpha^{4} \beta^{6} d_{8} \\
& +20 \alpha^{3} \beta^{6} c_{4}+20 \alpha^{3} \beta^{6} d_{3}-20 \alpha^{3} \beta^{6} d_{5}+80 \alpha^{2} \beta^{8} c_{2}+80 \alpha^{2} \beta^{8} d_{1} \\
& -32 \alpha^{2} \beta^{7} c_{2}-56 \alpha^{2} \beta^{7} c_{7}+56 \alpha^{2} \beta^{7} c_{9}-32 \alpha^{2} \beta^{7} d_{1}-56 \alpha^{2} \beta^{7} d_{6}
\end{aligned}
$$




$$
\begin{aligned}
& +56 \alpha^{2} \beta^{7} d_{8}-4 \alpha^{2} \beta^{6} c_{7}+4 \alpha^{2} \beta^{6} c_{9}-4 \alpha^{2} \beta^{6} d_{6}+4 \alpha^{2} \beta^{6} d_{8}+32 \beta^{9} c_{2} \\
& +32 \beta^{9} d_{1} \text {, } \\
& P_{17}=16 c_{7} \alpha^{18}-48 c_{9} \alpha^{18}-32 d_{8} \alpha^{18}+32 \beta c_{4} \alpha^{17}+32 \beta d_{3} \alpha^{17}-80 \beta^{2} c_{2} \alpha^{16} \\
& -72 \beta c_{7} \alpha^{16}-104 \beta c_{9} \alpha^{16}-64 \beta^{2} d_{1} \alpha^{16}-96 \beta d_{6} \alpha^{16}-80 \beta d_{8} \alpha^{16} \\
& +304 \beta^{2} c_{4} \alpha^{15}+240 \beta^{2} d_{3} \alpha^{15}-80 \beta^{2} d_{5} \alpha^{15}-488 \beta^{3} c_{2} \alpha^{14} \\
& -488 \beta^{2} c_{7} \alpha^{14}+136 \beta^{2} c_{9} \alpha^{14}-352 \beta^{3} d_{1} \alpha^{14}-384 \beta^{2} d_{6} \alpha^{14} \\
& +32 \beta^{2} d_{8} \alpha^{14}+808 \beta^{3} c_{4} \alpha^{13}+560 \beta^{3} d_{3} \alpha^{13}-280 \beta^{3} d_{5} \alpha^{13} \\
& -948 \beta^{4} c_{2} \alpha^{12}-758 \beta^{3} c_{7} \alpha^{12}+450 \beta^{3} c_{9} \alpha^{12}-624 \beta^{4} d_{1} \alpha^{12} \\
& -504 \beta^{3} d_{6} \alpha^{12}+196 \beta^{3} d_{8} \alpha^{12}+800 \beta^{4} c_{4} \alpha^{11}+500 \beta^{4} d_{3} \alpha^{11} \\
& -300 \beta^{4} d_{5} \alpha^{11}-680 \beta^{5} c_{2} \alpha^{10}-405 \beta^{4} c_{7} \alpha^{10}+295 \beta^{4} c_{9} \alpha^{10} \\
& -400 \beta^{5} d_{1} \alpha^{10}-240 \beta^{4} d_{6} \alpha^{10}+130 \beta^{4} d_{8} \alpha^{10}+260 \beta^{5} c_{4} \alpha^{9} \\
& +150 \beta^{5} d_{3} \alpha^{9}-100 \beta^{5} d_{5} \alpha^{9}-192 \beta^{6} c_{2} \alpha^{8}-58 \beta^{5} c_{7} \alpha^{8}+46 \beta^{5} c_{9} \alpha^{8} \\
& -112 \beta^{6} d_{1} \alpha^{8}-30 \beta^{5} d_{6} \alpha^{8}+18 \beta^{5} d_{8} \alpha^{8}+32 \beta^{6} c_{4} \alpha^{7}+48 \beta^{6} d_{3} \alpha^{7} \\
& -16 \beta^{6} d_{5} \alpha^{7}-288 \beta^{7} c_{2} \alpha^{6}+16 \beta^{6} c_{2} \alpha^{6}+32 \beta^{6} c_{7} \alpha^{6}-64 \beta^{6} c_{9} \alpha^{6} \\
& -320 \beta^{7} d_{1} \alpha^{6}+32 \beta^{6} d_{1} \alpha^{6}+24 \beta^{6} d_{6} \alpha^{6}-56 \beta^{6} d_{8} \alpha^{6}+232 \beta^{7} c_{4} \alpha^{5} \\
& -48 \beta^{6} c_{4} \alpha^{5}+312 \beta^{7} d_{3} \alpha^{5}-80 \beta^{6} d_{3} \alpha^{5}-72 \beta^{7} d_{5} \alpha^{5}-944 \beta^{8} c_{2} \alpha^{4} \\
& +160 \beta^{7} c_{2} \alpha^{4}+192 \beta^{7} c_{7} \alpha^{4}+52 \beta^{6} c_{7} \alpha^{4}-408 \beta^{7} c_{9} \alpha^{4}+20 \beta^{6} c_{9} \alpha^{4} \\
& -992 \beta^{8} d_{1} \alpha^{4}+192 \beta^{7} d_{1} \alpha^{4}+72 \beta^{7} d_{6} \alpha^{4}+72 \beta^{6} d_{6} \alpha^{4}-288 \beta^{7} d_{8} \alpha^{4} \\
& +360 \beta^{8} c_{4} \alpha^{3}-128 \beta^{7} c_{4} \alpha^{3}+440 \beta^{8} d_{3} \alpha^{3}-120 \beta^{7} d_{3} \alpha^{3}-40 \beta^{8} d_{5} \alpha^{3} \\
& -960 \beta^{9} c_{2} \alpha^{2}+160 \beta^{8} c_{2} \alpha^{2}+136 \beta^{8} c_{7} \alpha^{2}+32 \beta^{7} c_{7} \alpha^{2}-328 \beta^{8} c_{9} \alpha^{2} \\
& -8 \beta^{7} c_{9} \alpha^{2}-896 \beta^{9} d_{1} \alpha^{2}+96 \beta^{8} d_{1} \alpha^{2}+24 \beta^{8} d_{6} \alpha^{2}+24 \beta^{7} d_{6} \alpha^{2} \\
& -216 \beta^{8} d_{8} \alpha^{2}+208 \beta^{9} c_{4} \alpha+240 \beta^{9} d_{3} \alpha-80 \beta^{9} d_{5} \alpha-384 \beta^{10} c_{2} \\
& -48 \beta^{9} c_{7}+16 \beta^{9} c_{9}-320 \beta^{10} d_{1}-48 \beta^{9} d_{6}+16 \beta^{9} d_{8} \text {, } \\
& P_{18}=16 \alpha^{18} c_{7}-64 \alpha^{18} c_{9}-32 \alpha^{18} d_{8}+32 \alpha^{17} \beta d_{5}-16 \alpha^{16} \beta^{2} c_{2}+24 \alpha^{16} \beta c_{7} \\
& -296 \alpha^{16} \beta c_{9}-176 \alpha^{16} \beta d_{8}+64 \alpha^{15} \beta^{2} c_{4}+160 \alpha^{15} \beta^{2} d_{5}-136 \alpha^{14} \beta^{3} c_{2} \\
& -104 \alpha^{14} \beta^{2} c_{7}-460 \alpha^{14} \beta^{2} c_{9}-352 \alpha^{14} \beta^{2} d_{8}+248 \alpha^{13} \beta^{3} c_{4} \\
& +280 \alpha^{13} \beta^{3} d_{5}-324 \alpha^{12} \beta^{4} c_{2}-254 \alpha^{12} \beta^{3} c_{7}-262 \alpha^{12} \beta^{3} c_{9} \\
& -308 \alpha^{12} \beta^{3} d_{8}+300 \alpha^{11} \beta^{4} c_{4}+200 \alpha^{11} \beta^{4} d_{5}-280 \alpha^{10} \beta^{5} c_{2} \\
& -165 \alpha^{10} \beta^{4} c_{7}-30 \alpha^{10} \beta^{4} c_{9}-110 \alpha^{10} \beta^{4} d_{8}+126 \alpha^{9} \beta^{5} c_{4}+32 \alpha^{9} \beta^{5} d_{3} \\
& +50 \alpha^{9} \beta^{5} d_{5}-160 \alpha^{8} \beta^{6} c_{2}-96 \alpha^{8} \beta^{6} d_{1}-102 \alpha^{8} \beta^{5} c_{7}+32 \alpha^{8} \beta^{5} c_{9} \\
& -96 \alpha^{8} \beta^{5} d_{6}+22 \alpha^{8} \beta^{5} d_{8}+256 \alpha^{7} \beta^{6} c_{4}+368 \alpha^{7} \beta^{6} d_{3}-48 \alpha^{7} \beta^{6} d_{5} \\
& -16 \alpha^{7} \beta^{5} c_{4}-32 \alpha^{7} \beta^{5} d_{3}-704 \alpha^{6} \beta^{7} c_{2}-768 \alpha^{6} \beta^{7} d_{1}+48 \alpha^{6} \beta^{6} c_{2} \\
& -420 \alpha^{6} \beta^{6} c_{7}+12 \alpha^{6} \beta^{6} c_{9}+64 \alpha^{6} \beta^{6} d_{1}-600 \alpha^{6} \beta^{6} d_{6}+104 \alpha^{6} \beta^{6} d_{8} \\
& +56 \alpha^{6} \beta^{5} c_{7}+96 \alpha^{6} \beta^{5} d_{6}+1080 \alpha^{5} \beta^{7} c_{4}+1264 \alpha^{5} \beta^{7} d_{3}-200 \alpha^{5} \beta^{7} d_{5} \\
& -144 \alpha^{5} \beta^{6} c_{4}-160 \alpha^{5} \beta^{6} d_{3}-2080 \alpha^{4} \beta^{8} c_{2}-1952 \alpha^{4} \beta^{8} d_{1} \\
& +240 \alpha^{4} \beta^{7} c_{2}-812 \alpha^{4} \beta^{7} c_{7}-212 \alpha^{4} \beta^{7} c_{9}+192 \alpha^{4} \beta^{7} d_{1}-1104 \alpha^{4} \beta^{7} d_{6} \\
& +96 \alpha^{4} \beta^{7} d_{8}+156 \alpha^{4} \beta^{6} c_{7}+144 \alpha^{4} \beta^{6} d_{6}+1592 \alpha^{3} \beta^{8} c_{4}+1600 \alpha^{3} \beta^{8} d_{3}
\end{aligned}
$$




$$
\begin{aligned}
& -280 \alpha^{3} \beta^{8} d_{5}-192 \alpha^{3} \beta^{7} c_{4}-120 \alpha^{3} \beta^{7} d_{3}-2208 \alpha^{2} \beta^{9} c_{2} \\
& -1792 \alpha^{2} \beta^{9} d_{1}+160 \alpha^{2} \beta^{8} c_{2}-624 \alpha^{2} \beta^{8} c_{7}-200 \alpha^{2} \beta^{8} c_{9}+64 \alpha^{2} \beta^{8} d_{1} \\
& -768 \alpha^{2} \beta^{8} d_{6}+56 \alpha^{2} \beta^{8} d_{8}+48 \alpha^{2} \beta^{7} c_{7}+24 \alpha^{2} \beta^{7} d_{6}+800 \alpha \beta^{9} c_{4} \\
& +720 \alpha \beta^{9} d_{3}-160 \alpha \beta^{9} d_{5}-832 \beta^{10} c_{2}-512 \beta^{10} d_{1}-192 \beta^{9} c_{7}+48 \beta^{9} c_{9} \\
& -144 \beta^{9} d_{6}+32 \beta^{9} d_{8} \\
& P_{19}=16 \alpha^{18} c_{9}+96 \alpha^{16} \beta c_{9}+212 \alpha^{14} \beta^{2} c_{9}+208 \alpha^{12} \beta^{3} c_{9}-16 \alpha^{10} \beta^{4} c_{7} \\
& +117 \alpha^{10} \beta^{4} c_{9}+32 \alpha^{10} \beta^{4} d_{8}-16 \alpha^{9} \beta^{5} c_{4}-32 \alpha^{9} \beta^{5} d_{3}-32 \alpha^{9} \beta^{5} d_{5} \\
& +64 \alpha^{8} \beta^{6} c_{2}+64 \alpha^{8} \beta^{6} d_{1}+8 \alpha^{8} \beta^{5} c_{7}+226 \alpha^{8} \beta^{5} c_{9}+96 \alpha^{8} \beta^{5} d_{6} \\
& +224 \alpha^{8} \beta^{5} d_{8}+16 \alpha^{8} \beta^{4} c_{7}-32 \alpha^{8} \beta^{4} c_{9}-32 \alpha^{8} \beta^{4} d_{8}-272 \alpha^{7} \beta^{6} c_{4} \\
& -288 \alpha^{7} \beta^{6} d_{3}-208 \alpha^{7} \beta^{6} d_{5}+32 \alpha^{7} \beta^{5} d_{5}+592 \alpha^{6} \beta^{7} c_{2}+448 \alpha^{6} \beta^{7} d_{1} \\
& -16 \alpha^{6} \beta^{6} c_{2}+496 \alpha^{6} \beta^{6} c_{7}+516 \alpha^{6} \beta^{6} c_{9}+528 \alpha^{6} \beta^{6} d_{6}+520 \alpha^{6} \beta^{6} d_{8} \\
& -16 \alpha^{6} \beta^{5} c_{7}-96 \alpha^{6} \beta^{5} c_{9}-96 \alpha^{6} \beta^{5} d_{8}-1176 \alpha^{5} \beta^{7} c_{4}-888 \alpha^{5} \beta^{7} d_{3} \\
& -424 \alpha^{5} \beta^{7} d_{5}+64 \alpha^{5} \beta^{6} c_{4}+80 \alpha^{5} \beta^{6} d_{5}+1792 \alpha^{4} \beta^{8} c_{2}+1088 \alpha^{4} \beta^{8} d_{1} \\
& -96 \alpha^{4} \beta^{7} c_{2}+1288 \alpha^{4} \beta^{7} c_{7}+532 \alpha^{4} \beta^{7} c_{9}+984 \alpha^{4} \beta^{7} d_{6}+488 \alpha^{4} \beta^{7} d_{8} \\
& -84 \alpha^{4} \beta^{6} c_{7}-72 \alpha^{4} \beta^{6} c_{9}-72 \alpha^{4} \beta^{6} d_{8}-1824 \alpha^{3} \beta^{8} c_{4}-1120 \alpha^{3} \beta^{8} d_{3} \\
& -320 \alpha^{3} \beta^{8} d_{5}+88 \alpha^{3} \beta^{7} c_{4}+40 \alpha^{3} \beta^{7} d_{5}+2048 \alpha^{2} \beta^{9} c_{2}+1024 \alpha^{2} \beta^{9} d_{1} \\
& -64 \alpha^{2} \beta^{8} c_{2}+1080 \alpha^{2} \beta^{8} c_{7}+200 \alpha^{2} \beta^{8} c_{9}+672 \alpha^{2} \beta^{8} d_{6}+176 \alpha^{2} \beta^{8} d_{8} \\
& -24 \alpha^{2} \beta^{7} c_{7}-8 \alpha^{2} \beta^{7} c_{9}-8 \alpha^{2} \beta^{7} d_{8}-944 \alpha \beta^{9} c_{4}-480 \alpha \beta^{9} d_{3} \\
& -80 \alpha \beta^{9} d_{5}+768 \beta^{10} c_{2}+256 \beta^{10} d_{1}+240 \beta^{9} c_{7}-16 \beta^{9} c_{9}+96 \beta^{9} d_{6} \\
& +16 \beta^{9} d_{8} \text {, } \\
& P_{20}=4 \alpha^{8} c_{7}-12 \alpha^{8} c_{9}-8 \alpha^{8} d_{8}+8 \alpha^{7} \beta d_{5}-4 \alpha^{6} \beta^{2} c_{2}+4 \alpha^{6} \beta c_{7}-62 \alpha^{6} \beta c_{9} \\
& -40 \alpha^{6} \beta d_{8}+4 \alpha^{6} c_{9}+16 \alpha^{5} \beta^{2} c_{4}+36 \alpha^{5} \beta^{2} d_{5}-32 \alpha^{4} \beta^{3} c_{2}-29 \alpha^{4} \beta^{2} c_{7} \\
& -99 \alpha^{4} \beta^{2} c_{9}-66 \alpha^{4} \beta^{2} d_{8}+6 \alpha^{4} \beta c_{9}+54 \alpha^{3} \beta^{3} c_{4}+50 \alpha^{3} \beta^{3} d_{5} \\
& -64 \alpha^{2} \beta^{4} c_{2}-48 \alpha^{2} \beta^{3} c_{7}-54 \alpha^{2} \beta^{3} c_{9}-38 \alpha^{2} \beta^{3} d_{8}+\alpha^{2} \beta^{2} c_{9} \\
& +44 \alpha \beta^{4} c_{4}+20 \alpha \beta^{4} d_{5}-32 \beta^{5} c_{2}-12 \beta^{4} c_{7}-6 \beta^{4} c_{9}-4 \beta^{4} d_{8} \text {, } \\
& P_{21}=32 \beta^{2} c_{9}\left(\alpha^{2}+2 \beta\right)^{3}\left(4 \alpha^{4}+6 \alpha^{2} \beta+\beta^{2}\right) \text {. }
\end{aligned}
$$

We claim that at least 15 of the functions $f_{0}, \ldots, f_{21}$ are linearly independent. Indeed we expand these functions up to twenty-first order in the variable $R$ around $R=0$, and we obtain that the rank of the coefficient matrix of $1, R, \ldots, R^{21}$ of the functions $f_{0}, \ldots, f_{21}$ is 15 . So the claim is proved.

The coefficients

$$
\begin{aligned}
B_{0}= & \frac{\pi}{4 \beta^{2}}\left(\left(a_{6}-a_{8}-b_{7}+b_{9}\right)+\left(c_{6}-c_{8}-d_{7}+d_{9}\right)\right), \\
B_{1}= & \frac{\pi}{2 \beta}\left(\left(a_{8}-b_{9}\right)-\left(c_{8}-d_{9}\right)\right), \\
B_{2}= & -\frac{\pi}{4 \beta}\left(\left(2 \beta a_{1}+2 \beta b_{2}-a_{6}+3 a_{8}+b_{7}-3 b_{9}\right)+\left(2 \beta c_{1}+2 \beta d_{2}-c_{6}\right.\right. \\
& \left.\left.+3 c_{8}+d_{7}-3 d_{9}\right)\right),
\end{aligned}
$$




$$
\begin{aligned}
B_{3}= & -\frac{\pi}{2}\left(\left(2 \alpha^{2} a_{1}+\alpha a_{3}+\alpha a_{5}+2 \beta a_{1}+a_{6}+a_{8}\right)+\left(2 \alpha^{2} c_{1}+\alpha c_{3}+\alpha c_{5}\right.\right. \\
& \left.\left.+2 \beta c_{1}+c_{6}+c_{8}\right)\right),
\end{aligned}
$$

are mutually linearly independent and are linearly independent with the remaining coefficients because in $B_{0}$ appears the parameter $b_{7}$ which does not appear in the coefficients $B_{j}$ for $j=4, \ldots, 21$, in $B_{1}$ appears the parameter $a_{8}$ which does not appear in the coefficients $B_{j}$ for $j=4, \ldots, 21$, in $B_{2}$ appears the parameter $a_{1}$ which does not appear in the coefficients $B_{j}$ for $j=4, \ldots, 21$, and in $B_{3}$ appears the parameter $a_{5}$ which does not appear in the coefficients $B_{j}$ for $j=4, \ldots, 21$. We write the coefficients $B_{4}, \ldots, B_{21}$ in the following matrix form

$$
\left(\begin{array}{c}
B_{4} \\
\vdots \\
B_{21}
\end{array}\right)=M v
$$

where $v=\left(a_{2}, a_{4}, a_{7}, a_{9}, b_{1}, b_{3}, b_{5}, b_{6}, b_{9}, c_{2}, c_{4}, c_{7}, c_{9}, d_{1}, d_{3}, d_{5}, d_{6}, d_{9}\right)$ and $M$ is an $18 \times 18$ matrix. Due to the size of the matrix $M$ we do not give it explicitly here. The calculations have been done with the algebraic manipulator Mathematica. The rank of $M$ is 9 . Thus there exist $4+9=13$ independent coefficients. So we can write

$$
f(R)=\sum_{i=0}^{21} f_{i} B_{i}=\sum_{j=0}^{12} \tilde{f}_{i} \widetilde{B}_{i} .
$$

Now we check the assumptions of Theorem 4. Note that $h(x, y)=y, \mathcal{M}=$ $\left\{(x, y) \in \mathbb{R}^{2}: y=0\right\}$, and system (12) can be written in the following way

$$
\frac{d R}{d \varphi}=Z(\varphi, R)=\varepsilon F(\varphi, R)+\mathcal{O}\left(\varepsilon^{2}\right)
$$

where

$$
\begin{aligned}
F(\varphi, R) & =F_{1}(\varphi, R)+\operatorname{sign}(h(\varphi, R)) F_{2}(\varphi, R) \\
F_{1}(\varphi, R) & =\frac{\mu\left(x^{2}+y^{2}\right)\left(\left(Q p_{1}-P q_{1}\right)+\left(Q p_{2}-P q_{2}\right)\right)}{4 R(Q x-P y)} \\
F_{2}(\varphi, R) & =\frac{\mu\left(x^{2}+y^{2}\right)\left(\left(Q p_{1}-P q_{1}\right)-\left(Q p_{2}-P q_{2}\right)\right)}{4 R(Q x-P y)}
\end{aligned}
$$

with $x=\rho(\varphi, R) \cos \varphi$ and $y=\rho(\varphi, R) \sin \varphi$. The assumptions (i), (ii) and (iii) of Theorem 4 are satisfied. In fact the assumption (i) is satisfied because $f$ is analytic in $R \in(0, \sqrt{-1 /(2 \beta)})$.

We note that a simple zero of a function of one variable always has nonzero Brouwer degree, for more details see [20]. So by (ii) we only need to look for the simple zeros of the function $f$ given in (13). 
The function $h(\varphi, R)=\rho(\varphi, R) \sin \varphi$ is equal to zero if and only if $\varphi=0$ or $\varphi=\pi$. Moreover we can check that $(d h / d \varphi)(0, R)=0$ if and only if

$$
\frac{\left(2 \beta R^{2}+1\right)\left(a^{2} R^{2}+2 b R^{2}+1\right)}{\sqrt{R^{2}\left(\alpha^{2}+2 \beta\right)+1}}+\alpha R\left(-2 \beta R^{2}-1\right)=0 .
$$

Again, we take the square in both sides of the equation obtained passing the second member to the right hand side of the previous equality and we obtain after some simplifications $\left(2 \beta R^{2}+1\right)^{3}=0$, and as before it is not possible. In a similar way it can be proved that $(d h / d \varphi)(\pi, R) \neq 0$. Thus the assumption (iii) is satisfied.

Since the assumptions of Theorem 4 are satisfied the simple zeros of the function (13) provide $2 \pi$-periodic solutions of system (12). Finally, by Proposition 6 it follows that the function $f$ given by (13) can be have at least 12 simple zeros. So Theorem 2 is proved.

\section{ACKNOWLEDGements}

The first author is partially supported by a MINECO/FEDER grant number MTM2009-03437, by an AGAUR grant number 2009SGR-410, by an ICREA Academia, two FP7+PEOPLE+2012+IRSES numbers 316338 and 318999, and FEDER-UNAB10-4E-378. The first and second author are supported by CAPES-MECD grant PHB-2009-0025-PC. The third author is supported by FAPESP-2010/17956-1.

\section{REFERENCES}

[1] V.I. Arnold and Y.S. Ilyashenko, Dynamical Systems I. Ordinary differential equations, Encyclopedia Math. Sci., vol 1, Springer, Berlin, 1988.

[2] T.R. Blows and L.M. Perko, Bifurcation of limit cycles from centers and separatrix cycles of planar analytic systems, SIAM Rev. 36 (1994), 341-376.

[3] A. Buică and J. Llibre, Averaging methods for finding periodic orbits via Brower degree, Bull. Sci. Math. 128 (2004), 7-22.

[4] J. Chavarriga, Integrable systems in the plane with a center type linear, Applicationes Mathematicae 22 (1996), 285-309.

[5] J. Chavarriga and I. Garcia, Isocrhonous centers of cubic reversible systems, Dynamical Systems, Plasmas and Gravitation (Orléans la Source, 1997), 255-268, Lecture Notes in Phis. 518, Springer, Berlin, 1999.

[6] J. Chavarriga and M. Sabatini, A survey of isochronous centers, Qual. Theory of Dyn. Syst. 1 (1999), 1-70.

[7] C. Chicone and M. Jacobs, Bifurcation of limit cycles from quadratic isochrones, J. Differential Equations 91 (1991), 268-326.

[8] C. Christopher and C. Li, Limit cycles in differential equations, Birkhauser, Boston, 2007.

[9] H. Giacomini, J. Llibre and M. Viano, On the nonexistence, existence and uniqueness of limit cycles, Nonlinearity 9 (1996), 501-516.

[10] H. Giacomini, J. Llibre and M. Viano, On the shape of limit cycles that bifurcate from Hamiltonian centers, Nonlinear Anal. 43 (2000), 523-537.

[11] H. Giacomini, J. Llibre and M. Viano, On the shape of limit cycles that bifurcate from non-Hamiltonian centers, Nonlinear Anal. 43 (2001), 837-859. 
[12] J. Guckenheimer and P. Holmes, Nonlinear oscillations, dynamical systems, and bifurcations of vector fields, Applied Mathematical Sciences, vol. 42, Springer-Verlag, New York, 1986.

[13] J. Karlin and W.J. Studden, T-Systems: With applications in analysis and statistics, Pure Appl. Math., Interscience Publishers, New York, London, Sidney, (1966).

[14] C. Li and W. Li, Linear estimation of the number of zeros of abelian integrals for some cubic isochronous centers, J. of Differential Equations 180 (2002), 307-333.

[15] J. Llibre, Averaging theory and limit cycles for quadratic systems, Rad. Mat. 11 (2002/3), 215-228.

[16] J. Llibre, A. Mahdi and J. Roé, The geometry of the real planar polynomial differential systems having their orbits embedded in conics, Dynamical Systems 3 (2011), 287-321.

[17] J. Llibre, A. Mahdi and N. Vulpe, Phase portraits and invariant straight lines of cubic polynomial vector fields having a quadratic rational first integral, Rocky Mountain J. of Math. 5 (2011), 1585-1629.

[18] J. Llibre, D.D. Novaes and M.A. Teixeira, Averaging methods for studying the periodic orbits of discontinuous differential systems, http://arxiv.org/pdf/1205.4211.pdf.

[19] J. Llibre and G. Świrszcz, On the limit cycles of polynomial vector fields, Dynamics of Continuous, Discrete and Impulsive Systems, Serie A 18 (2011), 203-214.

[20] N.G. Lloyd, Degree theory, Cambridge University Press, 1978.

[21] W.S. Loud, Behavior of the period of solutions of certain plane autonomous systems near centers, Contributions to Differential Equations 3 (1964), 21-36.

[22] L.S. Pontrjagin, Über Autoschwingungssysteme, die den hamiltonshen nahe liegen, Physikalische Zeitschrift der Sowjetunion 6 (1934), 25-28.

[23] J.A. Sanders, F. Verhulst and J. Murdock, Averaging methods in nonlinear dynamical systems, Applied Mathematical Sci., vol. 59, Springer, New York, 2007.

[24] F. Verhulst, Nonlinear differential equations and dynamical systems, Universitext, Springer, 1991.

[25] M. Viano, J. Llibre and H.Giacomini, Arbitrary order bifurcations for perturbed Hamiltonian planar systems via the reciprocal of an integrating factor, Nonlinear Anal. 48 (2002), 117-136.

1 Departament de Matemàtiques, Universitat Autònoma de Barcelona, 08193 Bellaterra, Barcelona, Catalonia, Spain.

E-mail address: jllibre@mat.uab.cat

E-mail address: blopes@mat.uab.cat

2 Departamento de Matemática - IBIlCe-UnesP, Rua C. Colombo, 2265, CEP 15054-000 S. J. Rio Preto, SÃo Paulo, Brazil.

E-mail address: jrezmoraes@gmail.com 